\title{
Yield response and economic implications of soybean (Glycine max (L.). Merrill) - lowland-upland rice sequential cropping in the rainforest/savanna transitory ecosystem
}

\author{
Paul Abayomi Sobowale SOREMI*1, Olalekan Sulaimon SAKARIYAWO ${ }^{1}$, Kehinde Adebayo OKELEYE ${ }^{1}$, Victor \\ Idowu OLOWE ${ }^{2}$, Jamiu Oladipupo AZEEZ ${ }^{3}$, Francis NWILENE ${ }^{4}$, Sunday Gbenga ADERIBIGBE ${ }^{1}$
}

Received June 27, 2017; accepted October 12, 2017.

Delo je prispelo 27. junija 2017, oktobra 12. avgusta 2017.

\begin{abstract}
A sequential cropping system of soybean-lowland (NERICA L-42)-upland (NERICA 2) rice was established at Abeokuta and Ibadan (Nigeria) to evaluate the performance of the cropping system. Field trials were in split-split plot arrangement fitted into randomised complete block design and replicated three times. The first sequence had in the main plot tillage [minimum (MT) and conventional (CT)]. Soybean varieties TGx 1448-2E (V1) and TGx 1740-2F (V2) in subplot and spacing in sub-sub plot were $60 \mathrm{~cm} \times 5 \mathrm{~cm}(\mathrm{R} 1)$, $60 \mathrm{~cm} \times 10(\mathrm{R} 2)$ and $60 \mathrm{~cm} \times 15 \mathrm{~cm}(\mathrm{R} 3)$. Individual rice plots were established by dry dibble (DD) seeding and transplanting and sub-sub plot spacing were $15 \mathrm{~cm} \times 15 \mathrm{~cm}$ (S1), $20 \mathrm{~cm} \times 20$ and $25 \mathrm{~cm}(\mathrm{~S} 2) \times 25 \mathrm{~cm}(\mathrm{~S} 3)$. The sequence soybean (V1 CT R3); lowland and upland rice (S3 CT DD) was the most economically efficient (N 1,754 $\mathrm{ha}^{-1}$ day $\left.^{-1}\right)$ in Abeokuta, while soybean (V1 MT R1) and lowland and upland rice (S1 DD MT) was the most economically efficient (N 1,858 ha $^{-1}$ day $^{-1}$ ) in Ibadan.
\end{abstract}

Key words: conventional tillage; dry dibble; economic efficiency; minimum tillage; NERICA rice; soybean varieties; spacing, transplanting

\section{IZVLEČEK}

\section{ODZIV PRIDELKA IN EKONOMIČNOST PRIDELAVE SOJE (Glycine max (L.). Merrill) V KOLOBARJU Z RIŽEM, PRILAGOJENEM GOJENJU V VODI IN RIŽEM, PRILAGOJENEM GOJENJU V SUHIH RAZMERAH V PREHODNIH EKOSISTEMIH, NASTALIH IZ DEŽEVNEGA PRAGOZDA IN SAVANE}

$\mathrm{Z}$ namenom ovrednotenja več pridelovalnih sistemov je bil v Abeokuti in Ibadanu (Nigeria) vzpostavljen kolobar soje z rižem, prilagojenem gojenju $\mathrm{v}$ vodi ('NERICA L-42') in rižem, prilagojenem gojenju v suhih razmerah ('NERICA 2'). Poskus je temeljil na popolnem naključnem bloku s tremi ponovitvami. Prvi kolobar je na glavnih površinah obsegal dva načina obdelave tal: minimalno (MT) in konvencionalno obdelavo (CT). Sorti soje TGx 1448-2E (V1) in TGx 1740-2F (V2) sta bili na podpovršinah posejani $\mathrm{v}$ gostotah $60 \times 5 \mathrm{~cm}$ (R1), $60 \times 10 \mathrm{~cm}(\mathrm{R} 2)$ in $60 \times 15 \mathrm{~cm}(\mathrm{R} 3)$. Posamezne površine $\mathrm{z}$ rižem so bile osnovane $\mathrm{z}$ neposredno setvijo $\mathrm{V}$ 'jamice' (DD) in s presajanjem sadik, gostote sklopa pa so bile $15 \times 15 \mathrm{~cm}(\mathrm{~S} 1), 20 \times 20 \mathrm{~cm}$ in $25(\mathrm{~S} 2) \times 25 \mathrm{~cm}(\mathrm{~S} 3)$. Kolobar $\mathrm{s}$ sojo (V1 CT R3) ter rižem, prilagojenem gojenju v vodi in rižem, prilagojenim gojenju v suhih razmerah (S3 CT DD) je bil ekonomsko najbolj učinkovit (N 1,754 ha hay $^{-1}$ ) v Abeokuti, medtem, ko je bil kolobar s sojo (V1 MT R1) ter rižem, prilagojenem gojenju $\mathrm{v}$ vodi in rižem, prilagojenem gojenju v suhih razmerah (S1 DD MT) ekonomsko najbolj učinkovit (N 1,858 ha $^{-1}$ day $^{-1}$ ) v Ibadanu.

Ključne besede: konvencilnalna obdelava tal; neposredna setev V jamice; ekonomska učinkovitost; minimalna obdelava tal; 'NERICA' sorte riža; sorte soje; gostota sklopa; presajanje riža

\footnotetext{
1 Department of Plant Physiology and Crop Production, Federal University of Agriculture, Abeokuta (FUNAAB), P.M.B. 2240, Abeokuta, Ogun State, Nigeria; * Corresponding author: elawob_ass@hotmail.com

2 Institute of Food Security, Environmental Resources and Agricultural Research, FUNAAB. P.M.B. 2240, Abeokuta, Ogun State, Nigeria

3 Department of Soil Science and Land Management, FUNAAB, P.M.B 2240, Abeokuta, Ogun State, Nigeria

4 Africa Rice Center (WARDA-Nigeria), \% IITA, Oyo Road, Ibadan, PMB 5320, Ibadan, Oyo State, Nigeria
} 


\section{INTRODUCTION}

Meeting the food and nutritional needs of the majority of the populace remains a major challenge to most government in sub-Sahara Africa (SSA). Rice is rapidly becoming a staple food item because of its ease of preparation for consumption. However, in Nigeria the annual consumption of $5.0 \mathrm{M} \mathrm{t}$ is far above the $3.0 \mathrm{M} \mathrm{t}$ production level (Daramola, 2005). The shortfall is met through massive importation despite dwindling foreign exchange. The situation calls for increased production. Increased production can be achieved through extensive or intensive cultivation. Competing needs for land due to increasing population (Chidumayo, 1987) and requirements for development purposes make extensive cultivation unattractive. Thus, intensive cultivation could provide solution to the challenge. Intensification of farming system has its own challenges. Increased use of agricultural input is beyond the reach of resource challenged farmers. One promising ameliorative measure is sequential cropping system that could ensure double or triple cropping of staple crops like rice in SSA.

Sequential cropping system involving rice must be conducted with the right mix of component crops and management practice to ensure sustainable production. Soybean is an important oilseed crop (Harold et al., 1990) and a potential component of cropping system to increase soil fertility through its capability of biologically fixing atmospheric nitrogen (Harold et al., 1990). Other management practices to ensure sustainable production of rice could be the tillage practice, seeding method and crop spacing. Conventional production of lowland rice involves puddling of the field (Farooq et al., 2011). This technique has its drawbacks such as modification of physico-chemical properties of submerged soil that could negatively affect plant growth and yield (Pande et al., 1985; Wade et al., 1998). Transplanting had been the major seeding method especially for lowland rice, but the cost of its establishment (Chan and Nor, 1993) and the problem of water use efficiency and conservation (Gill et al., 2006) remain its major drawbacks. Rice that is directly seeded had been proposed in the past to address this problem (AmpongNyarko, 1996; Fischer and Antigua, 1996; Rao et al., 2007). However, the prevalence of weed (Morris, 1990; Rao et al., 2007), panicle sterility (Farooq et al., 2009) and nutrient availability (Farooq et al., 2011) had reduced its adoption by most farmers. Increasing plant density to the optimum could ensure maximum utilization of growth resources. This would provide the major crop a more competitive edge against the incidence of weeds that was observed in directly seeded rice. This observation was made on maize by Mashingaidze, (2004) and Zimdahl, (2013).

There is very little information in the literature on the agronomic performance of soybean introduced into the cropping sequence in a lowland ecosystem. Hence there is need to know the appropriateness of soybean in soybean-lowland rice-upland rice sequential cropping and its economic implication in rainforest Savanna agroecology. The objectives of the trials were to evaluate grain yield response of soybean and NERICA rice cultivars to tillage and spacing; determine responses of NERICA rice cultivars to seeding methods in the inland valley in soybean-lowland-upland rice cropping system. The trials also aimed to determine productivity of rice-based sequential cropping and its economic consequences in the rainforest/savanna transitory ecology.

\section{MATERIALS AND METHODS}

\subsection{Description of the Study Site}

Field trials were established at rainfed inland valley of FUNAAB (latitude $7^{\circ} 15^{\prime} \mathrm{N}$ and longitude $3^{\circ} 25^{\prime} \mathrm{E}$; and altitude $76 \mathrm{~m}$ above sea level), and at paddy F14 at the Research Farm of Africa Rice Centre, Ibadan station (latitude $7^{0} 30^{\prime} \mathrm{N}$ and longitude $3^{0} 54^{\prime} \mathrm{E}$ ), International
Institute of Tropical Agriculture (IITA) premises, Oyo State, Nigeria. At Abeokuta, the total monthly rainfall was between $288.1 \mathrm{~mm}$ (October, 2011) to absence of precipitation (November, December, 2011 and January, 2012). Mean monthly temperature was in the range $29.2{ }^{\circ} \mathrm{C}$ (April, 2011) to $24.5^{\circ} \mathrm{C}$ (July, 2011) (Table 1). 
Yield response and economic implications of soybean ... cropping in the rainforest/savanna transitory ecosystem

Table 1: Rainfall and temperature patterns, Abeokuta

\begin{tabular}{ccccccccccccccc}
\hline Parameters & Units & $\begin{array}{c}\text { Jan. } \\
\text { Total }\end{array}$ & $\begin{array}{c}\text { Feb. } \\
\text { '11 }\end{array}$ & $\begin{array}{c}\text { Mar. } \\
\text { '11 }\end{array}$ & $\begin{array}{c}\text { Apr. } \\
\text { '11 }\end{array}$ & $\begin{array}{c}\text { May } \\
\text { '11 }\end{array}$ & $\begin{array}{c}\text { June } \\
\text { '11 }\end{array}$ & $\begin{array}{c}\text { July } \\
\text { '11 }\end{array}$ & $\begin{array}{c}\text { Aug. } \\
\text { '11 }\end{array}$ & $\begin{array}{c}\text { Sept. } \\
\text { '11 }\end{array}$ & $\begin{array}{c}\text { Oct. } \\
\text { '11 }\end{array}$ & $\begin{array}{c}\text { Nov. } \\
\text { '11 }\end{array}$ & $\begin{array}{c}\text { Dec. } \\
\text { '11 }\end{array}$ & $\begin{array}{c}\text { Jan. } \\
\text { '12 }\end{array}$ \\
\hline $\begin{array}{c}\text { Rainfall } \\
\text { Mean }\end{array}$ & $\mathrm{mm}$ & 0.00 & 139.8 & 23.9 & 74.5 & 73.7 & 84.5 & 349.5 & 88.7 & 204.1 & 288.1 & 3.6 & 0.00 & 0.00 \\
$\begin{array}{c}\text { Temp. } \\
{ }^{\circ} \mathrm{C}\end{array}$ & 27.2 & 28.9 & 29.2 & 29.2 & 28.0 & 26.9 & 24.5 & 25.3 & 26.6 & 26.9 & 27.9 & 27.0 & 27.0 \\
\hline
\end{tabular}

Table 2: Rainfall and temperature pattern, Ibadan

\begin{tabular}{|c|c|c|c|c|c|c|c|c|c|c|c|c|c|c|}
\hline Parameters & Units & $\begin{array}{c}\text { Jan. } \\
' 11\end{array}$ & $\begin{array}{c}\text { Feb. } \\
' 11\end{array}$ & $\begin{array}{c}\text { Mar. } \\
\text { '11 }\end{array}$ & $\begin{array}{l}\text { Apr. } \\
\text { '11 }\end{array}$ & $\begin{array}{c}\text { May } \\
\text { '11 }\end{array}$ & $\begin{array}{c}\text { June } \\
' 11\end{array}$ & $\begin{array}{c}\text { July } \\
' 11\end{array}$ & $\begin{array}{c}\text { Aug. } \\
' 11\end{array}$ & $\begin{array}{c}\text { Sept. } \\
' 11\end{array}$ & $\begin{array}{l}\text { Oct. } \\
' 11\end{array}$ & $\begin{array}{c}\text { Nov. } \\
' 11\end{array}$ & $\begin{array}{c}\text { Dec. } \\
' 11\end{array}$ & $\begin{array}{l}\text { Jan. } \\
\text { '12 }\end{array}$ \\
\hline $\begin{array}{c}\text { Total } \\
\text { Rainfall }\end{array}$ & $\mathrm{mm}$ & 0.00 & 130.6 & 72.3 & 103 & 143.1 & 224.4 & 156.4 & 314.9 & 280.9 & 262.4 & 8 & 0.00 & 0.00 \\
\hline $\begin{array}{l}\text { Mean } \\
\text { Temp. }\end{array}$ & ${ }^{\circ} \mathrm{C}$ & 26.2 & 28.3 & 28.8 & 28.0 & 27.6 & 26.5 & 25.0 & 24.5 & 25.9 & 25.9 & 27.7 & 26.6 & 26.7 \\
\hline
\end{tabular}

Total monthly rainfall pattern at Ibadan ranges was between $314.9 \mathrm{~mm}$ (August, 2011) to absence of precipitation (January and December, 2011 and January, 2012). Mean temperature was between $28.8{ }^{\circ} \mathrm{C}$ (March, 2011) to $24.5^{\circ} \mathrm{C}$ (August, 2011) (Table 2).

\subsection{Treatments and experimental design}

Sequential cropping system was conducted concurrently at two locations, which commenced from January 2011. The first crop in the sequence was soybean (Glycine $\max$ (L.). Merrill) varieties TGx 1448-2E (late maturing) and TGx 1740-2F (early maturing), and Telfairia occidentalis Hook. F (fluted pumpkin) in the control plot. Lowland rice 'NERICA ${ }^{\circledR}$ L-42' was established in the second sequence in June 2011. This variety is early maturing with maturity range of between 90 to 100 days. 'NERICA ${ }^{\circledR} 2$ ' an upland rice variety with maturity range of between 90 to 100 days was established as the third crop in the sequence between September and October 2011 in Abeokuta and Ibadan respectively. The experiments were established in a split-split plot arrangement fitted to randomised complete block design with three replicates in 2011/ 2012 cropping season. All experiments had tillage (conventional and minimum) in the main plot. For the soybean trial the main plot size was $33.5 \mathrm{~m} \times 26.5 \mathrm{~m}$. The sub-plot size was $13.5 \mathrm{~m} \times 13 \mathrm{~m}$, which consisted soybean variety (TGx 1448-2E and TGx 1740-2F) and sub-sub plot size measured $5 \mathrm{~m} \times 4 \mathrm{~m}$, consisted of plant spacing $(60 \mathrm{~cm} \times 5 \mathrm{~cm}, 60 \mathrm{~cm} \times 10 \mathrm{~cm}$ and $60 \mathrm{~cm}$ $\times 15 \mathrm{~cm}$ ). Net plot size was $9.6 \mathrm{~m}^{2}$. For the rice trials, the sub-plot measured $13.5 \mathrm{~m} \times 13 \mathrm{~m}$. Individual rice plots were established by dry direct seeding and transplanting. The sub-sub plot was $5 \mathrm{~m} \times 4 \mathrm{~m}$ had spacing $(15 \mathrm{~cm} \times 15 \mathrm{~cm}, 20 \mathrm{~cm} \times 20 \mathrm{~cm}$ and $25 \mathrm{~cm} \times$ $25 \mathrm{~cm}$ ). The control plot in the sequences was Telfairialowland-upland rice. Telfairia occidentalis was transplanted and land prepared for conventional tillage on mounds at $1 \mathrm{~m} \times 1 \mathrm{~m} \mathrm{(20} \mathrm{plants}$ plot $^{-1}$ and 10,000 plants $\left.\mathrm{ha}^{-1}\right)$, with the application of N P K 20:10:10 at

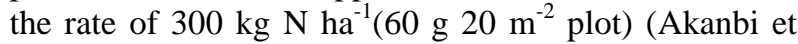
al., 2007). For the rice trials (both lowland and upland crops) plots were prepared conventionally and

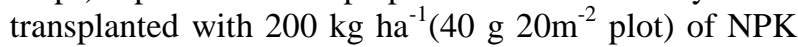
15:15:15 as basal application at transplanting and 65.2

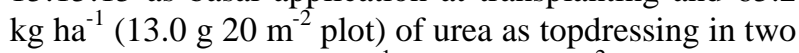

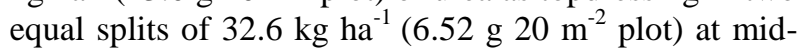
tillering (4 WAT/7 WAP) and panicle initiation (6 WAT/9 WAS). Soybean stover ranged between 2.96 to $6.70 \mathrm{~kg} \mathrm{plot}^{-1}$ and lowland rice straws of between 5.41 and $6.96 \mathrm{tha}^{-1}$ of $10 \mathrm{~cm}$ in lengths were incorporated into the soil and left for two weeks before planting succeeding crop to allow for decomposition and mineralisation. Dried poultry dropping was incorporated into to all the soybean plots two weeks before sowing at the rate of $16.6 \mathrm{~kg} \mathrm{plot}^{-1}$ and $2.1 \mathrm{~kg} \mathrm{plot}^{-1}(8.3$ and $1.03 \mathrm{t}$ $\mathrm{ha}^{-1}$ ) for Abeokuta and Ibadan respectively as suggested by Azeez \& Van Averbeke, (2010). This translated to $40 \mathrm{~g} \mathrm{~N}$ plot $^{-1}\left(20 \mathrm{~kg} \mathrm{~N} \mathrm{ha}^{-1}\right)$. The poultry droppings were sourced from layers pen under battery cage system.

\subsection{Land preparation}

Plots were laid out with an alley of $0.5 \mathrm{~m}$ between plots and $1 \mathrm{~m}$ between replicates. For soybean establishment minimum and conventional tillage methods were achieved manually (hand hoe), however, the intensity of soil disturbance was increased in conventional tillage through pulverization (small hand hoe and cutlass) of clods into smaller fragments. For rice establishment minimum tillage was achieved through reduced disturbance of soil manually (hand hoe), while conventional tillage was conducted manually (hoe, feet and hand) but with increased intensity of soil disturbance until it turned into slurry for reduced infiltration and percolation of water (puddling). Seeds for the soybean varieties were sourced from Institute of 
Food Security, Environmental Resources and Agriculture Research (IFSERAR) of FUNAAB, while those of the rice varieties were sourced from Africa Rice Centre, Ibadan sub-station. The fruit of the fluted pumpkin was locally sourced. Fluted pumpkin seedlings were raised in a nursery for three weeks before they were transplanted to the field. A dry bed nursery was established at the beginning of each rice cycle near the field. The size of the bed was $1 \mathrm{~m} \times 5 \mathrm{~m}$. The top soil was softened and watered. Rice seeds were sown on the date of seeding the dry direct seeded on the field and watered regularly for 3 weeks after which they were transplanted. The nursery of the lowland rice was established on $1^{\text {th }}$ and $20^{\text {th }}$ June 2011 at Abeokuta and Ibadan, respectively. While that of upland rice was established on $27^{\text {th }}$ September, and $5^{\text {th }}$ October, 2011 at Abeokuta and Ibadan, respectively.

\subsection{Planting Operations}

Three seeds of soybean per hole were planted on $15^{\text {th }}$ January, 2011 in Abeokuta and 20 ${ }^{\text {th }}$ January, 2011 at Ibadan at spacing's corresponding to the treatment combination, which was later thinned to two plants per stand at two weeks after sowing. The population of the fluted pumpkin was 10,000 plants per hectare at one seedling per stand. The vines of the fluted pumpkin were trained to climb platforms erected to facilitate its creeping habit.

The lowland rice trials were established in Abeokuta on 1st June, 2011 and at Ibadan on 7th June, 2011. The upland rice trials were established on 27th September, 2011 at Abeokuta and 5th October, 2011 at Ibadan. For the direct seeded, three seeds were sown and thinned to two seedlings per stand two weeks after sowing, while two 3 weeks old seedlings were transplanted for the transplanted treatment.

Weeding in soybean field was done manually (hoe) at 3 , 6 , and 10 weeks after planting (WAP). In rice plots Riceforce $^{\circledR}$ (a selective pre-emergence herbicide) with oxidiaxon as active ingredient at $0.25 \mathrm{~kg} \mathrm{l}^{-1}$ was applied on the day of establishing the direct seeded plots at the rate of $3 \mathrm{~kg}$ a.i. per hectare while OrizoPlus ${ }^{\circledR}$ (360 g of propanil and $200 \mathrm{~g}$ of 2, 4-D acid a.i./litre), a selective post-emergence herbicide was applied at the average recommended rate of $10 \mathrm{~kg}$ a.i. per hectare at $18 \mathrm{DAT}$. Off-type rice varieties were selectively removed manually during the life cycle of the rice.

\subsection{Sampling and measurements}

Pre-planting soil physico-chemical properties were determined for each crop cycle. A composite soil samples were randomly taken from a depth of 0-20 cm. Rice grain yield and yield components was determined at harvest maturity from ten representative hills per net plot. Leaf fresh mass of fluted pumpkins was determined at two-week intervals after four weeks of transplanting up to the $16^{\text {th }}$ week.

Productivity of the cropping system was evaluated based on total productivity of the sequence, production efficiency, economic efficiency, land utilization index, profitability, returns (gross and net), benefit and cost ratio. To evaluate the productivity of rice in the sequence, productivity of paddy was converted to milled rice (National Cereals Research Institute, 1992), while productivity of non-rice component in the sequence was converted to rice grain equivalent yield on price basis.

Rice grain equivalent yield (RGEY)=yield of non rice crop $\times$ price of non rice crop/price of rice (Manjunath \& Korikanthimah, 2004).

Prices as at third quarter 2011, of milled imported rice was N $202.34 \mathrm{~kg}^{-1}$ (market price of 'NERICA ${ }^{\circledR}$ ' was assumed to be equal to those of imported milled rice (Adigbo et al., 2010), and soybean was $\mathrm{N} 180.00 \mathrm{~kg}^{-1}$. This was obtained from Ogun State Agricultural Development Programe (OGADEP), Abeokuta, Ogun State. Farm gate price of fluted pumpkin (N100 kg ${ }^{-1}$ ) was used for computation. Production efficiency was computed by dividing the total grain production $\left(\mathrm{ha}^{-1}\right)$ in a sequence with total duration (days) of crops in a sequence (Tomar et al., 1990). Benefit: cost ratio was calculated for the different practices in each sequence by diving the net returns by the cost of cultivation per sequence (Prasad et al., 2011).

Land utilisation index $=(\mathrm{Ta}+\mathrm{Tb}+\mathrm{Tc}) / 365 \times 100$ (Tomar et al., 1990);

where; $\mathrm{Ta}, \mathrm{Tb}$ and $\mathrm{Tc}$ represent total duration (days) of crops $\mathrm{a}, \mathrm{b}$ and $\mathrm{c}$ in the sequence

Profitability $=$ net returns per hectare in the sequence $/ 365$ days (Prasad et al., 2011).

Economic efficiency $(\mathrm{EE})=\mathrm{Net}$ returns of sequence (Naira per hectare)/duration of sequence (days) (Patil et al., 1995). Duration indicated the sum total of the number of days all crops in a sequence attained maturity.

Total grain production is the sum total of all grain in a sequence, i.e. rice grain equivalent yield (RGEY) plus milled rice equivalent (MRE) of lowland and upland rice. Gross return was the total income from the sales of all the crops in a sequence i.e. price of milled rice multiplied by total grain production $\left(\mathrm{kg} \mathrm{ha}^{-1}\right)$. Net return was gross return less cost of cultivation. Cost of cultivation indicated the amount of money incurred in the producing all the crops in each sequence based on 
different operations performed and materials used for raising the crops in each sequence.

\subsection{Statistical Analysis}

Data collected were subjected to mixed model Analysis of Variance (ANOVA) using the GenStat $12^{\text {th }}$ Edition and the differences among treatment means were separated using least significant difference (LSD) at $5 \%$ probability level where $\mathrm{F}$ values were significant.

\section{RESULTS}

Before the establishment of soybean in the inland valley of Abeokuta, the soil $\mathrm{pH}$ was 6.50 , with organic content of $51.4 \mathrm{~g} \mathrm{~kg}^{-1}$. The nutrient composition was $1.80 \mathrm{~g} \mathrm{~kg}^{-1}$ (total nitrogen), and $6.91 \mathrm{mg} \mathrm{kg} \mathrm{kg}^{-1}$ (available phosphorus). The textural class was loamy sand. At the commencement of lowland rice, soil $\mathrm{pH}$ was 6.75 , with organic content of $53.1 \mathrm{~g} \mathrm{~kg}^{-1}$. The nutrient composition was $0.90 \mathrm{~g} \mathrm{~kg}^{-1}$ (total nitrogen), and $10.69 \mathrm{mg} \mathrm{kg}^{-1}$ (available phosphorus). The textural class was sandy loam. Soil property as at the time of establishing upland rice was soil $\mathrm{pH} 6.95$, with organic content of $58.8 \mathrm{~g} \mathrm{~kg}^{-}$ 1. The nutrient composition was $2.50 \mathrm{~g} \mathrm{~kg}^{-1}$ (total nitrogen), and $10.76 \mathrm{mg} \mathrm{kg}^{-1}$ (available phosphorus).
The textural class was sandy loam. Soil pH at the establishment of soybean at Ibadan was 5.50, while soil organic content and total nitrogen was $32.2 \mathrm{~g} \mathrm{~kg}^{-1}$ and $1.90 \mathrm{~g} \mathrm{~kg}^{-1}$, respectively. The soil was sandy clay loam. Prior to the establishment of lowland rice, the soil $\mathrm{pH}$ was close to neutral (6.85), with organic matter content of $49.10 \mathrm{~g} \mathrm{~kg}^{-1}$. Nutrient composition was: $0.60 \mathrm{~g} \mathrm{~kg}^{-1}$ total nitrogen and $4.49 \mathrm{mg} \mathrm{kg}^{-1}$ available $\mathrm{P}$; the textural class was the same as that of soybean establishment. Soil properties at the start of upland rice in the sequence were $7.05 \mathrm{pH}, 66.70 \mathrm{~g} \mathrm{~kg}^{-1}$ organic matter and $2.90 \mathrm{~g} \mathrm{~kg}^{-}$ ${ }^{1}$ total nitrogen. Available $\mathrm{P}$ was $8.1 \mathrm{mg} \mathrm{kg}^{-1}$. The soil was sandy loam (Table 3).

Table 3: Soil physico-chemical properties of the experimental site, in soybean-lowland-upland-rice at Abeokuta and Ibadan

\begin{tabular}{|c|c|c|c|c|c|c|c|}
\hline \multirow[b]{2}{*}{$\begin{array}{c}\text { Parameters and methods of } \\
\text { determination }\end{array}$} & & \multicolumn{3}{|c|}{ ABEOKUTA } & \multicolumn{3}{|c|}{ IBADAN } \\
\hline & Units & Soil A & Soil B & Soil C & Soil A & Soil B & Soil C \\
\hline $\begin{array}{c}\mathrm{pH} \text { (in water, 1:1),pH meter } \\
\text { (McLean, 1982) }\end{array}$ & - & 6.50 & 6.75 & 6.95 & 5.50 & 6.85 & 7.05 \\
\hline $\begin{array}{c}\text { Organic matter, Walkey- } \\
\text { Black method (Allison, } \\
\text { 1965) }\end{array}$ & $\mathrm{g} \mathrm{kg}^{-1}$ & 51.4 & 53.1 & 58.8 & 32.2 & 49.1 & 66.7 \\
\hline $\begin{array}{c}\text { Total nitrogen, modified } \\
\text { micro Kjeldahl method } \\
\text { (Jackson, 1962) }\end{array}$ & $\mathrm{g} \mathrm{kg}^{-1}$ & 1.80 & 0.90 & 2.50 & 1.90 & 0.60 & 2.90 \\
\hline $\begin{array}{c}\text { Available phosphorus (Bray } \\
\text { and Kurtz, 1945) }\end{array}$ & $\mathrm{mg} \mathrm{kg}^{-1}$ & 6.91 & 10.69 & 10.76 & 6.52 & 4.49 & 8.1 \\
\hline \multicolumn{8}{|l|}{$\begin{array}{c}\text { Exchangeable, flame } \\
\text { photometry }\end{array}$} \\
\hline - Potassium & $\mathrm{cmol} \mathrm{kg}^{-1}$ & 0.21 & 0.24 & 0.11 & 0.71 & 0.41 & 0.09 \\
\hline - $\quad$ Sodium & $\mathrm{cmol} \mathrm{kg}^{-1}$ & 0.5 & 0.62 & 0.20 & 0.16 & 0.84 & 0.22 \\
\hline \multicolumn{8}{|l|}{$\begin{array}{c}\text { Particle size (Bouyoucos, } \\
1962)\end{array}$} \\
\hline - $\quad$ Sand & $\mathrm{g} \mathrm{kg}^{-1}$ & 778 & 734 & 750 & 580 & 544 & 730 \\
\hline - Clay & $\mathrm{g} \mathrm{kg}^{-1}$ & 56 & 125 & 120 & 220 & 245 & 130 \\
\hline - $\quad$ Silt & $\mathrm{g} \mathrm{kg}^{-1}$ & 166 & 141 & 130 & 200 & 211 & 140 \\
\hline Textural class & - & $\begin{array}{c}\text { Loamy } \\
\text { sand }\end{array}$ & $\begin{array}{l}\text { Sandy } \\
\text { loam }\end{array}$ & $\begin{array}{l}\text { Sandy } \\
\text { loam }\end{array}$ & $\begin{array}{l}\text { Sandy } \\
\text { clay loam }\end{array}$ & $\begin{array}{l}\text { Sandy } \\
\text { clay loam }\end{array}$ & $\begin{array}{l}\text { Sandy } \\
\text { loam }\end{array}$ \\
\hline
\end{tabular}

Legend: A - At soybean establishment, B - At lowland rice establishment, C - At upland rice establishment, \% - percent, $\mathrm{cmol} \mathrm{kg}^{-1}$ - centimoles of cations per kilogram, $\mathrm{mg} \mathrm{kg}^{-1}$ - milligramme kilogramme ${ }^{-1}, \mathrm{~g} \mathrm{~kg}^{-1}-$ gramme kilogramme $^{-1}$ 


\subsection{Grain yield and yield components of soybean at Abeokuta and Ibadan}

Tillage practices had no significant effect on all the yield components and grain yield of soybean in Abeokuta (Table 4). Similar trend was observed in Ibadan (Table 5). Soybean variety TGx 1448-2E had significantly $(P<0.05)$ higher mass of pod plot $^{-1}$, mass of seed plot $^{-1}$ and grain yield than cultivar TGx 1740-2F in Abeokuta, except mass of seed pod $^{-1}$ where, soybean cultivar TGx 1740-2F was observed to have significantly $(P<0.05)$ higher mass of seed pod ${ }^{-1}$ than cultivar TGx 1448-2E. However, in Ibadan there was no significant varietal variability on all the yield components and grain yield. In Abeokuta and Ibadan increasing plant density resulted in a significant $(P<0.05)$ increase in stover mass plot $^{-1}$. However, a converse trend was observed on 100 seed mass (Abeokuta) and mass of seed pod $^{-1}$ (Ibadan).

Table 4: Effect of tillage and row spacing on the yield components and grain yield of soybean cultivars in the inland valley at Abeokuta, 2011

\begin{tabular}{|c|c|c|c|c|c|c|c|c|c|c|}
\hline $\begin{array}{l}\text { Tillage } \\
\text { (T 1df) }\end{array}$ & $\begin{array}{l}\text { Mass of } \\
\text { pod plot }^{-1} \\
(\mathrm{~g})\end{array}$ & $\begin{array}{l}\text { Mass of } \\
\text { pod plant } \\
1(\mathrm{~g})\end{array}$ & $\begin{array}{l}\text { Mass of } \\
\text { seed plot } \\
{ }^{1}(\mathrm{~g})\end{array}$ & $\begin{array}{l}\text { Mass of } \\
\text { seed plant } \\
{ }^{1}(\mathrm{~g})\end{array}$ & $\begin{array}{l}\text { Mass of } \\
\text { seed pod } \\
(\mathrm{g})\end{array}$ & $\begin{array}{l}\text { Threshing } \\
\text { percentage } \\
(\%)\end{array}$ & $\begin{array}{l}\text { Stover } \\
\text { mass plot } \\
{ }^{1}(\mathrm{~kg})\end{array}$ & $\begin{array}{l}100 \\
\text { seed } \\
\text { mass } \\
(\mathrm{g})\end{array}$ & $\begin{array}{l}\text { Harvest } \\
\text { index }\end{array}$ & $\begin{array}{l}\text { Grain } \\
\text { yield }(\mathrm{kg} \\
\left.\mathrm{ha}^{-1}\right)\end{array}$ \\
\hline Minimum & 1602 & 26.28 & 822.9 & 4.026 & 0.264 & 51.89 & 4.49 & 11.21 & 44.4 & 772.9 \\
\hline Conventional & 1520 & 29.94 & 784.8 & 4.292 & 0.274 & 51.90 & 4.34 & 10.54 & 46.7 & 737.6 \\
\hline $\operatorname{LSD}(0.05)$ & 347.4 & 19.745 & 146.82 & 0.9701 & 0.0518 & 3.075 & 1.174 & 0.683 & 18.92 & 138.79 \\
\hline \multicolumn{11}{|l|}{$\begin{array}{l}\text { Variety } \\
\text { (V 1df) }\end{array}$} \\
\hline TGx $1448-2 \mathrm{E}$ & 1651 & 27.35 & 836.0 & 3.968 & 0.263 & 51.00 & 4.31 & 11.08 & 44.1 & 785.2 \\
\hline TGx1740-2F & 1471 & 28.87 & 771.7 & 4.349 & 0.275 & 52.79 & 4.53 & 10.67 & 47.0 & 725.3 \\
\hline $\operatorname{LSD}(0.05)$ & $152.4^{*}$ & 3.580 & $33.70 *$ & 0.7042 & $0.0409^{*}$ & 3.720 & 0.601 & 0.793 & 9.41 & $32.66^{*}$ \\
\hline \multicolumn{11}{|l|}{$\begin{array}{l}\text { Spacing } \\
\text { (S 2df) }\end{array}$} \\
\hline $\begin{array}{l}60 \mathrm{~cm} \times 5 \\
\mathrm{~cm}\end{array}$ & 1590 & 30.35 & 804.9 & 4.210 & 0.175 & 50.84 & 6.35 & 7.28 & 48.3 & 756.6 \\
\hline $\begin{array}{l}60 \mathrm{~cm} \times 10 \\
\mathrm{~cm}\end{array}$ & 1556 & 28.25 & 796.5 & 4.122 & 0.266 & 51.61 & 3.89 & 11.09 & 46.0 & 747.8 \\
\hline $\begin{array}{l}60 \mathrm{~cm} \times 15 \\
\mathrm{~cm}\end{array}$ & 1539 & 25.73 & 810.2 & 4.144 & 0.367 & 53.23 & 3.02 & 14.25 & 42.4 & 761.4 \\
\hline $\operatorname{LSD}(0.05)$ & 138.4 & 3.793 & 28.93 & 0.3521 & 0.0542 & 5.043 & $0.673 *$ & $0.972 *$ & 7.05 & 26.98 \\
\hline $\mathrm{T} \times \mathrm{V}(1 \mathrm{df})$ & Ns & Ns & Ns & Ns & Ns & Ns & Ns & Ns & Ns & Ns \\
\hline $\mathrm{T} \times \mathrm{S}(2 \mathrm{df})$ & Ns & Ns & Ns & $*$ & Ns & Ns & Ns & Ns & Ns & Ns \\
\hline$V \times S(2 d f)$ & Ns & Ns & Ns & Ns & Ns & Ns & Ns & Ns & Ns & Ns \\
\hline $\begin{array}{l}T \times V \times S \\
(2 d f)\end{array}$ & Ns & Ns & Ns & Ns & Ns & Ns & Ns & Ns & Ns & Ns \\
\hline
\end{tabular}

Legend: * - significant at $5 \%$ level, ** - significant at $1 \%$ level. 
Yield response and economic implications of soybean ... cropping in the rainforest/savanna transitory ecosystem

Table 5: Effect of tillage and row spacing on the yield components and grain yield of soybean cultivars in the inland valley at Ibadan, 2011

\begin{tabular}{|c|c|c|c|c|c|c|c|c|c|c|}
\hline Tillage (T 1df) & $\begin{array}{l}\text { Mass of } \\
\text { pod } \\
\text { plot }^{-1} \\
(\mathrm{~g})\end{array}$ & $\begin{array}{l}\text { Mass of } \\
\text { pod } \\
\text { plant }^{-1} \\
(\mathrm{~g})\end{array}$ & $\begin{array}{l}\text { Mass of } \\
\text { seed } \\
\text { plot }^{-1} \\
(\mathrm{~g})\end{array}$ & $\begin{array}{l}\text { Mass of } \\
\text { seed } \\
\text { plant }^{-1} \\
(\mathrm{~g})\end{array}$ & $\begin{array}{l}\text { Mass of } \\
\text { seed pod }^{-1} \\
(\mathrm{~g})\end{array}$ & $\begin{array}{l}\text { Threshing } \\
\text { percentage } \\
(\%)\end{array}$ & $\begin{array}{l}\text { Stover } \\
\text { mass } \\
\operatorname{plot}^{-1}(\mathrm{~kg})\end{array}$ & $\begin{array}{l}100 \\
\text { seed } \\
\text { mass } \\
(\mathrm{g})\end{array}$ & $\begin{array}{l}\text { Harvest } \\
\text { index }\end{array}$ & $\begin{array}{l}\text { Grain } \\
\text { yield }(\mathrm{kg} \\
\left.\mathrm{ha}^{-1}\right)\end{array}$ \\
\hline Minimum & 1706 & 19.51 & 843 & 4.14 & 0.32 & 49.60 & 4.26 & 14.70 & 43.4 & 793 \\
\hline Conventional & 1655 & 18.22 & 827 & 3.86 & 0.27 & 49.84 & 4.72 & 12.05 & 38.9 & 777 \\
\hline LSD (0.05) & 141.9 & 6.200 & 258.5 & 0.535 & 0.058 & 12.49 & 2.019 & 6.058 & 11.57 & 243.5 \\
\hline \multicolumn{11}{|l|}{ Variety (V 1df) } \\
\hline TGx1448-2E & 1723 & 19.07 & 874 & 3.75 & 0.30 & 50.97 & 4.36 & 12.89 & 38.8 & 821 \\
\hline TGx1740-2F & 1638 & 18.66 & 796 & 4.25 & 0.29 & 48.47 & 4.62 & 13.86 & 43.5 & 749 \\
\hline LSD (0.05) & 292.1 & 5.282 & 114.0 & 0.514 & 0.060 & 4.825 & 0.364 & 2.628 & 6.63 & 104.8 \\
\hline \multicolumn{11}{|l|}{ Spacing (S 2df) } \\
\hline $60 \mathrm{~cm} \times 5 \mathrm{~cm}$ & 1690 & 19.20 & 868 & 4.21 & 0.27 & 51.27 & 6.79 & 12.66 & 44.7 & 816 \\
\hline $60 \mathrm{~cm} \times 10 \mathrm{~cm}$ & 1705 & 18.67 & 870 & 4.05 & 0.30 & 51.40 & 3.72 & 14.30 & 41.9 & 820 \\
\hline $60 \mathrm{~cm} \times 15 \mathrm{~cm}$ & 1646 & 18.72 & 766 & 3.74 & 0.33 & 46.48 & 2.96 & 13.17 & 36.9 & 720 \\
\hline LSD (0.05) & 176.8 & 3.489 & 108.4 & 0.635 & $0.0354 *$ & 4.800 & $0.816 *$ & 2.096 & 6.32 & 103.2 \\
\hline$T \times V(1 d f)$ & Ns & Ns & Ns & Ns & Ns & Ns & Ns & Ns & Ns & Ns \\
\hline $\mathrm{T} \times \mathrm{S}(2 \mathrm{df})$ & Ns & Ns & Ns & Ns & Ns & Ns & Ns & Ns & Ns & Ns \\
\hline $\mathrm{V} \times \mathrm{S}(2 \mathrm{df})$ & Ns & Ns & Ns & Ns & Ns & Ns & Ns & Ns & Ns & Ns \\
\hline $\mathrm{T} \times \mathrm{V} \times \mathrm{S}(2 \mathrm{df})$ & Ns & Ns & Ns & Ns & Ns & Ns & Ns & Ns & Ns & Ns \\
\hline
\end{tabular}

Legend: * - significant at $5 \%$ level.

\subsection{Grain yield and yield components of lowland rice ('NERICA L-42') at Abeokuta and Ibadan}

Rice grown under conventional tillage performed better (significant at $P<0.05$ ) than minimum tillage only in Abeokuta on panicle mass (Table 6). There were no significant differences between the tillage practices among other yield and yield component parameters examined. Dry dibble method resulted in significantly higher $(P<0.05)$ numbers of grains per panicle in
Abeokuta than the transplanted rice, while there were no significant differences on other yield components at both locations (Tables 6 and 7). Stover mass increased significantly $(P<0.05)$ with lower plant densities at both locations, conversely at Ibadan number of panicles per $\mathrm{m}^{2}$ increased significantly $(P<0.05)$ with increasing plant density. There were no significant differences among other reproductive parameters with varying plant densities.

Table 6: Effect of tillage, seeding method and spacing on reproductive growth parameters of lowland rice ('NERICA ${ }^{\circledR}$ L-42') grown during the main season after soybean in the inland valley at Abeokuta in 2011

\begin{tabular}{|c|c|c|c|c|c|c|c|c|}
\hline Treatments & $\begin{array}{c}\text { Panicle } \\
\text { mass (g) }\end{array}$ & $\begin{array}{c}\text { Panicle } \\
\text { length }(\mathrm{cm})\end{array}$ & $\begin{array}{l}\text { Number of } \\
\text { panicles } \mathrm{m}^{-2}\end{array}$ & $\begin{array}{c}\text { Number of } \\
\text { grains } \\
\text { panicle }^{-1}\end{array}$ & $\begin{array}{c}1000 \text { seed } \\
\text { mass }(\mathrm{g})\end{array}$ & $\begin{array}{c}\text { Harvest } \\
\text { Index }(\%)\end{array}$ & $\begin{array}{l}\text { Grain } \\
\text { Yield (t } \\
\left.\text { ha }^{-1}\right)\end{array}$ & $\begin{array}{l}\text { Stover mass } \\
\qquad\left(\mathrm{t} \mathrm{ha}^{-1}\right)\end{array}$ \\
\hline \multicolumn{9}{|l|}{ Tillage (T 1df) } \\
\hline Minimum & 18.81 & 25.66 & 138 & 135 & 24.97 & 42.55 & 3.03 & 6.00 \\
\hline Conventional & 21.44 & 26.57 & 139 & 128 & 25.54 & 44.78 & 3.05 & 5.96 \\
\hline LSD (0.05) & 2.30 & 2.73 & 36.64 & 18.08 & 3.73 & 14.51 & 0.78 & 0.42 \\
\hline \multicolumn{9}{|c|}{ Seeding Method ( $1 \mathrm{df}$ ) } \\
\hline Dry Dibble & 19.62 & 25.50 & 138 & 136 & 25.67 & 43.74 & 3.04 & 5.94 \\
\hline Transplanted & 20.63 & 26.73 & 139 & 127 & 24.84 & 43.59 & 3.04 & 6.01 \\
\hline $\operatorname{LSD}(0.05)$ & 1.09 & 2.71 & 27.21 & 21.39 & 3.68 & 5.60 & 0.13 & 0.48 \\
\hline \multicolumn{9}{|l|}{ Spacing (S 2df) } \\
\hline $15 \times 15$ & 19.87 & 26.03 & 143 & 130 & 24.75 & 42.49 & 3.01 & 5.41 \\
\hline $20 \times 20$ & 19.81 & 25.88 & 136 & 136 & 25.51 & 42.25 & 3.07 & 6.00 \\
\hline $25 \times 25$ & 20.70 & 26.43 & 137 & 129 & 25.50 & 46.27 & 3.05 & 6.52 \\
\hline $\operatorname{LSD}(0.05)$ & 1.08 & 2.22 & 16.91 & 12.28 & 2.29 & 4.69 & 0.33 & 0.45 \\
\hline \multicolumn{9}{|l|}{ INTERACTIONS } \\
\hline $\mathrm{T} \times \mathrm{M}(1 \mathrm{df})$ & ns & ns & ns & ns & ns & ns & ns & ns \\
\hline $\mathrm{T} \times \mathrm{S}(2 \mathrm{df})$ & ns & ns & ns & ns & ns & ns & ns & ns \\
\hline $\mathrm{M} \times \mathrm{S}(2 \mathrm{df})$ & $*$ & ns & $*$ & ns & ns & ns & ns & ns \\
\hline $\mathrm{T} \times \mathrm{M} \times \mathrm{S}(2 \mathrm{df})$ & $*$ & ns & ns & ns & ns & ns & ns & ns \\
\hline
\end{tabular}

Legend: $\mathrm{g}$ - gramme(s), cm - centimetre(s), $\mathrm{m}^{2}$ - square metre(s), \% - percent, tha - tonnes per hectare, ns - not significant, LSD - Least significant differences of means (5\% level), T - Tillage, S - Spacing, M - Seeding method, * - significant at $5 \%$ level. 
Paul Abayomi Sobowale SOREMI et al.

Table 7: Effect of tillage, seeding method and spacing on reproductive growth parameters of lowland rice ('NERICA ${ }^{\mathbb{B}}$ L-42') grown during the main season in the inland valley at Ibadan in 2011

\begin{tabular}{|c|c|c|c|c|c|c|c|c|}
\hline Treatments & $\begin{array}{l}\text { Panicle } \\
\text { mass (g) }\end{array}$ & $\begin{array}{c}\text { Panicle } \\
\text { length }(\mathrm{cm})\end{array}$ & $\begin{array}{l}\text { Number of } \\
\text { panicle } \mathrm{m}^{-2}\end{array}$ & $\begin{array}{c}\text { Number of } \\
\text { grains } \\
\text { panicle }^{-1}\end{array}$ & $\begin{array}{l}1000 \text { seed } \\
\text { mass }(\mathrm{g})\end{array}$ & $\begin{array}{c}\text { Harvest } \\
\text { index }(\%)\end{array}$ & $\begin{array}{c}\text { Grain } \\
\text { yield (t } \\
\left.\text { ha }^{-1}\right)\end{array}$ & $\begin{array}{l}\text { Stover mass } \\
\left(\mathrm{t} \mathrm{ha}^{-1}\right)\end{array}$ \\
\hline \multicolumn{9}{|l|}{ Tillage (T 1df) } \\
\hline Minimum & 15.61 & 23.66 & 141 & 123 & 25.76 & 43.0 & 2.9 & 6.30 \\
\hline Conventional & 15.31 & 25.03 & 138 & 143 & 26.53 & 49.0 & 3.18 & 6.58 \\
\hline LSD (0.05) & 4.21 & 1.80 & 35.53 & 33.55 & 0.85 & 14.75 & 1.23 & 0.93 \\
\hline \multicolumn{9}{|l|}{$\begin{array}{c}\text { Seeding Method (M } \\
1 \mathrm{df})\end{array}$} \\
\hline Dry Dibble & 15.01 & 23.62 & 139 & 133 & 25.98 & 45.2 & 3.06 & 6.53 \\
\hline Transplanted & 15.91 & 25.07 & 140 & 133 & 26.31 & 46.8 & 3.01 & 6.35 \\
\hline $\operatorname{LSD}(0.05)$ & 2.34 & 1.79 & 22.21 & 20.16 & 2.97 & 5.50 & 0.47 & 0.55 \\
\hline \multicolumn{9}{|l|}{ Spacing (S 2df) } \\
\hline $15 \times 15$ & 14.55 & 24.88 & 161.4 & 133.0 & 27.79 & 45.0 & 2.75 & 6.00 \\
\hline $20 \times 20$ & 15.72 & 23.56 & 128.3 & 136.4 & 25.56 & 45.1 & 3.12 & 6.37 \\
\hline $25 \times 25$ & 16.12 & 24.60 & 129.0 & 130.1 & 25.09 & 48.0 & 3.24 & 6.96 \\
\hline LSD (0.05) & 1.76 & 1.66 & 17.08 & 16.32 & 1.99 & 5.97 & 0.40 & 0.40 \\
\hline \multicolumn{9}{|l|}{ INTERACTIONS } \\
\hline $\mathrm{T} \times \mathrm{M}(1 \mathrm{df})$ & ns & ns & ns & ns & ns & ns & ns & ns \\
\hline $\mathrm{T} \times \mathrm{S}(2 \mathrm{df})$ & ns & ns & ns & ns & ns & ns & ns & ns \\
\hline $\mathrm{M} \times \mathrm{S}(2 \mathrm{df})$ & ns & $* *$ & ns & $*$ & ns & ns & ns & ns \\
\hline $\mathrm{T} \times \mathrm{M} \times \mathrm{S}(2 \mathrm{df})$ & ns & ns & ns & ns & ns & ns & ns & ns \\
\hline
\end{tabular}

\subsection{Grain yield and yield components of upland rice ('NERICA 2') at Abeokuta and Ibadan}

There were no significant differences at both location between the tillage practices on yield and yield components (Tables 8 and 9). Dry dibble method seeded rice plants produced significantly higher numbers of panicles per $\mathrm{m}^{2}$ in Abeokuta than the transplanted rice plant. Similar pattern was observed on the number of grains per panicles at Ibadan. Reduced plant density at Ibadan resulted in a significant increase $(P<0.05)$ in panicle mass, number of grains per panicle and grain yield. However, at Abeokuta none of the yield components was significantly affected by plant densities.

Table 8: Effects of tillage, seeding method and spacing on reproductive growth parameters of upland rice ('NERICA ${ }^{\circledR} 2$ ') grown after lowland rice during the late season in the inland valley at Abeokuta in 2011

\begin{tabular}{|c|c|c|c|c|c|c|c|}
\hline Treatments & $\begin{array}{l}\text { Panicle mass } \\
\text { (g) }\end{array}$ & $\begin{array}{l}\text { Panicle length } \\
(\mathrm{cm})\end{array}$ & $\begin{array}{l}\text { Number of } \\
\text { panicles } \mathrm{m}^{-2}\end{array}$ & $\begin{array}{c}\text { Number of } \\
\text { grains panicle } \\
\text { grain }^{-}\end{array}$ & $\begin{array}{l}1000 \text { seed } \\
\text { mass }(g)\end{array}$ & $\begin{array}{l}\text { Harvest index } \\
(\%)\end{array}$ & $\begin{array}{c}\text { Grain yield (t } \\
\left.\text { ha }^{-1}\right)\end{array}$ \\
\hline \multicolumn{8}{|l|}{ Tillage (T 1df) } \\
\hline Minimum & 14.84 & 21.60 & 87 & 93 & 23.80 & 59.4 & 1.62 \\
\hline Conventional & 14.59 & 22.36 & 89 & 92 & 23.61 & 60.2 & 1.76 \\
\hline LSD (0.05) & 5.69 & 0.67 & 7.53 & 11.89 & 5.31 & 6.62 & 0.83 \\
\hline \multicolumn{8}{|c|}{$\begin{array}{c}\text { Seeding Method (M } \\
1 \mathrm{df})\end{array}$} \\
\hline Dry Dibble & 14.85 & 21.96 & 90 & 93 & 24.24 & 59.9 & 1.75 \\
\hline Transplanted & 14.58 & 22.01 & 86 & 91 & 23.17 & 59.7 & 1.64 \\
\hline LSD (0.05) & 1.36 & 3.03 & 4.00 & 3.87 & 1.66 & 3.23 & 0.11 \\
\hline \multicolumn{8}{|l|}{ Spacing (S 2df) } \\
\hline $15 \times 15$ & 14.56 & 22.01 & 87 & 93 & 23.57 & 60.5 & 1.68 \\
\hline $20 \times 20$ & 14.32 & 21.80 & 87 & 91 & 24.54 & 59.5 & 1.66 \\
\hline $25 \times 25$ & 15.28 & 22.13 & 90 & 93 & 23.01 & 59.5 & 1.75 \\
\hline LSD (0.05) & 1.46 & 1.40 & 6.24 & 6.87 & 2.32 & 5.61 & 0.20 \\
\hline \multicolumn{8}{|l|}{ INTERACTIONS } \\
\hline $\mathrm{T} \times \mathrm{M}(1 \mathrm{df})$ & ns & ns & ns & ns & ns & ns & $*$ \\
\hline $\mathrm{T} \times \mathrm{S}(2 \mathrm{df})$ & ns & $*$ & $*$ & ns & ns & ns & ns \\
\hline $\mathrm{M} \times \mathrm{S}(2 \mathrm{df})$ & ns & ns & ns & ns & ns & ns & ns \\
\hline $\mathrm{T} \times \mathrm{M} \times \mathrm{S}(2 \mathrm{df})$ & ns & ns & ns & ns & ns & ns & ns \\
\hline
\end{tabular}

Legend: $\mathrm{g}$ - gramme(s), cm - centimetre(s), $\mathrm{m}^{2}-$ square metre(s), \% - percent, $\mathrm{t} \mathrm{ha}^{-1}$ - tonnes per hectare, ns - not significant, LSD - Least significant differences of means (5\% level), T - Tillage, S - Spacing, M - Seeding method, * - significant at $5 \%$ level. 
Yield response and economic implications of soybean ... cropping in the rainforest/savanna transitory ecosystem

Table 9: Effects of tillage, seeding method and spacing on reproductive growth parameters of upland rice ('NERICA ${ }^{\circledR} 2$ ') grown after lowland rice during the late season in the inland valley at Ibadan in 2011

\begin{tabular}{|c|c|c|c|c|c|c|c|}
\hline Treatments & $\begin{array}{l}\text { Panicle mass } \\
\text { (g) }\end{array}$ & $\begin{array}{l}\text { Panicle length } \\
(\mathrm{cm})\end{array}$ & $\begin{array}{c}\text { Number of } \\
\text { panicles } \mathrm{m}^{-2}\end{array}$ & $\begin{array}{c}\text { Number of } \\
\text { grains panicle }\end{array}$ & $\begin{array}{l}1000 \text { seed } \\
\text { mass }(\mathrm{g})\end{array}$ & $\begin{array}{l}\text { Harvest index } \\
(\%)\end{array}$ & $\begin{array}{c}\text { Grain yield } \\
\text { (t/ha) }\end{array}$ \\
\hline \multicolumn{8}{|l|}{ Tillage (T 1df) } \\
\hline Minimum & 12.68 & 21.65 & 92 & 98 & 23.19 & 62.0 & 1.73 \\
\hline Conventional & 12.25 & 21.79 & 91 & 99 & 23.75 & 61.8 & 1.67 \\
\hline LSD (0.05) & 1.36 & 2.28 & 46.18 & 5.64 & 3.80 & 12.60 & 0.26 \\
\hline \multicolumn{8}{|c|}{$\begin{array}{c}\text { Seeding Method (M } \\
1 \mathrm{df})\end{array}$} \\
\hline Dry Dibble & 12.54 & 21.81 & 96 & 100.7 & 23.70 & 61.8 & 1.70 \\
\hline Transplanted & 12.39 & 21.63 & 87 & 95.3 & 23.24 & 62.0 & 1.70 \\
\hline LSD $(0.05)$ & 1.46 & 1.66 & 12.36 & 5.34 & 1.88 & 5.76 & 0.11 \\
\hline \multicolumn{8}{|l|}{ Spacing (S 2df) } \\
\hline $15 \times 15$ & 11.58 & 21.24 & 89 & 89 & 22.95 & 62.9 & 1.50 \\
\hline $20 \times 20$ & 12.26 & 22.05 & 89 & 97 & 23.86 & 60.9 & 1.77 \\
\hline $25 \times 25$ & 13.56 & 21.88 & 96 & 108 & 23.61 & 61.9 & 1.84 \\
\hline $\operatorname{LSD}(0.05)$ & 1.04 & 1.73 & 10.94 & 5.60 & 1.79 & 7.80 & 0.26 \\
\hline \multicolumn{8}{|l|}{ INTERACTIONS } \\
\hline $\mathrm{T} \times \mathrm{M}(1 \mathrm{df})$ & ns & ns & ns & ns & ns & ns & $*$ \\
\hline $\mathrm{T} \times \mathrm{S}(2 \mathrm{df})$ & ns & ns & $*$ & ns & ns & ns & $\mathrm{ns}$ \\
\hline $\mathrm{M} \times \mathrm{S}(2 \mathrm{df})$ & ns & $\mathrm{ns}$ & $\mathrm{ns}$ & ns & $\mathrm{ns}$ & $\mathrm{ns}$ & $\mathrm{ns}$ \\
\hline $\mathrm{T} \times \mathrm{M} \times \mathrm{S}(2 \mathrm{df})$ & ns & $\mathrm{ns}$ & $\mathrm{ns}$ & ns & ns & $\mathrm{ns}$ & $\mathrm{ns}$ \\
\hline
\end{tabular}

\subsection{Economic productivity and profitability of soybean-lowland rice-upland rice}

At Abeokuta, productivity of fluted pumpkin $\left(0.99 \mathrm{t}^{\mathrm{C}} \mathrm{a}^{-}\right.$ ${ }^{1}$ ) expressed as rice grain equivalent yield (RGEY) was the highest $(P<0.05)$, while the least significant RGEY $\left(0.62 \mathrm{t} \mathrm{ha}^{-1}\right)$ was observed when soybean 'TGx 1448$2 \mathrm{E}^{\prime}$ was cultivated under conventional tillage at the spacing of $60 \mathrm{~cm} \times 5 \mathrm{~cm}$. Conversely, for lowland and upland rice in the sequence the least significant productivity was observed in the plots preceded by fluted pumpkin under conventional tillage practises, transplanted at a spacing of $20 \mathrm{~cm} \times 20 \mathrm{~cm}$. The highest milled lowland rice productivity $\left(2.50 \mathrm{t} \mathrm{ha}^{-1}\right)$ was observed when directly seeded under conventional tillage at the least plant population density $(25 \mathrm{~cm} \times$ $25 \mathrm{~cm})$. However, the highest $(P<0.05)$ milled rice productivity $\left(1.47 \mathrm{t} \mathrm{ha}^{-1}\right)$ of upland rice was observed under conventional tillage, transplanted at the spacing of $15 \mathrm{~cm} \times 15 \mathrm{~cm}$. Sequence that consisted of soybean 'TGx 1448-2E', 'NERICA L-42' and 'NERICA 2' under conventional tillage, at the spacing of $60 \mathrm{~cm} \times$ $15 \mathrm{~cm}$ and $25 \mathrm{~cm} \times 25 \mathrm{~cm}$ (soybean and rice cultivars respectively) recorded the highest total productivity $\left(4.57 \mathrm{t} \mathrm{ha}^{-1}\right)$ and production efficiency $\left(15.69 \mathrm{~kg} \mathrm{ha}^{-1}\right.$ day $\left.^{-1}\right)$. The sequence that consisted of fluted pumpkin, 'NERICA L-42' and 'NERICA 2' cultivated under conventional tillage that was transplanted at a spacing of $20 \mathrm{~cm} \times 20 \mathrm{~cm}$ had significantly the least total productivity (3.69 $\left.\mathrm{t} \mathrm{ha}^{-1}\right)$, production efficiency $\left(11.40 \mathrm{~kg} \mathrm{ha}^{-1}\right.$ day $\left.^{-1}\right)$, profitability $\left(\mathrm{N} 729 \mathrm{ha}^{-1} \mathrm{day}^{-1}\right)$ and conversely the highest land utilization index $(88.58 \%)$. The highest $(P<0.05)$ profitability $\left(\mathrm{N} 1404\right.$ ha $^{-1}$ day $^{-1)}$ was observed in the sequence that consisted of soybean 'TGx 1448-2E', under minimum tillage, at a spacing of
$60 \mathrm{~cm} \times 15 \mathrm{~cm}$ and $25 \mathrm{~cm} \times 25 \mathrm{~cm}$ in soybean, lowland and upland rice respectively with the least plant population densities. The least land utilization index $(77.63 \%)$ was observed in the sequence that consisted of soybean 'TGx 1448-2E', under minimum tillage, at a spacing of $60 \mathrm{~cm} \times 10 \mathrm{~cm}$ and $20 \mathrm{~cm} \times 20 \mathrm{~cm}$ in soybean, lowland and upland rice respectively (Table $10)$.

At Ibadan, the highest RGEY (1.04 $\left.\mathrm{t} \mathrm{ha}^{-1}\right)$ was observed when the fluted pumpkin was established in the control plot preceding rice in the sequence. However, the least RGEY $\left(0.5 \mathrm{t} \mathrm{ha}^{-1}\right)$ was observed when soybean 'TGx 1448 -2E' was established under minimum tillage at the spacing of $60 \mathrm{~cm} \times 5 \mathrm{~cm}$. As observed in Abeokuta, the treatment combinations that had the least significant productivity of milled lowland and upland rice was also observed at Ibadan. Similar pattern of cropping sequence and treatment combinations were observed on the least total productivity, production efficiency, profitability and the highest LUI was repeated at Ibadan. The highest productivity $\left(2.70 \mathrm{tha}^{-1}\right)$ of milled lowland rice was observed when it was transplanted and established under conventional tillage at the spacing of $25 \mathrm{~cm} \times 25 \mathrm{~cm}$. In upland rice, the highest productivity of milled rice was obtained when 'NERICA 2' was cultivated under minimum tillage and directly seeded at the spacing of $15 \mathrm{~cm} \times 15 \mathrm{~cm}$. The highest total productivity $\left(4.75 \mathrm{t} \mathrm{ha}^{-1}\right)$ was achieved in the sequence when soybean 'TGx 1740-2F', lowland and upland rice cultivars were established under minimum tillage, with both rice cultivars directly seeded at the spacing of $25 \mathrm{~cm} \times 25 \mathrm{~cm}$, while soybean was spaced at $60 \mathrm{~cm} \times$ $15 \mathrm{~cm}$. Similar cropping sequence gave the highest 
production efficiency $\left(15.84 \mathrm{~kg} \mathrm{ha}^{-1} \mathrm{day}^{-1}\right)$. The highest profitability (1531 ha hay $^{-1}$ ) was observed in the sequence of soybean 'TGx 1448-2E' at the spacing $60 \mathrm{~cm} \times 5 \mathrm{~cm}$, lowland and upland rice directly seeded at the spacing $15 \mathrm{~cm} \times 15 \mathrm{~cm}$ under minimum tillage. Cultivar TGx 1448-2E at a spacing of $60 \mathrm{~cm} \times 10 \mathrm{~cm}$, succeeded by lowland and upland rice at a spacing of $20 \mathrm{~cm} \times 20 \mathrm{~cm}$, directly seeded gave the least LUI $(77.63 \%)$ (Table 11).

The least significant net returns, benefit/cost ratio and economic efficiency at both locations was recorded in the sequence fluted pumpkin, lowland and upland land rice that was transplanted, established under conventional tillage at a spacing of $20 \mathrm{~cm} \times 20 \mathrm{~cm}$. The sequence of 'TGx 1448-2E' soybean at the spacing $60 \mathrm{~cm} \times 15 \mathrm{~cm}$, lowland and upland rice at the spacing of $25 \mathrm{~cm} \times 25 \mathrm{~cm}$ directly seeded and established under minimum tillage in Abeokuta produced the highest net returns (N 512, 488). At Ibadan, the sequence of ' $T G x$ $1448-2 \mathrm{E}$ ' soybean at the spacing of $60 \mathrm{~cm} \times 5 \mathrm{~cm}$ and lowland and upland rice at a spacing of $15 \mathrm{~cm} \times 15 \mathrm{~cm}$ that was directly seeded and established under minimum tillage resulted in the highest net return ( $N$ 558,647). Similar pattern of treatment combinations in the cropping sequence was observed on the highest benefit/cost ratio at both locations. In Abeokuta soybean 'TGx 1448-2E' conventionally established at the $60 \mathrm{~cm}$ $\times 15 \mathrm{~cm}$ spacing, lowland and upland rice at a spacing of $25 \mathrm{~cm} \times 25 \mathrm{~cm}$ that were conventionally tilled and directly seeded was the most economically efficient ( $N$ $1,754 \mathrm{ha}^{-1} \mathrm{day}^{-1}$ ). The sequence of 'TGx 1448-2E' at the $60 \mathrm{~cm} \times 5 \mathrm{~cm}$ spacing, lowland and upland rice at a spacing of $15 \mathrm{~cm} \times 15 \mathrm{~cm}$ all crops minimally tilled and directly seeded was the most economically efficient ( $\mathrm{N}$ 1858 ha $^{-1}$ day $^{-1}$ ) at Ibadan (Table 12).

Table 10: Total productivity, duration, production efficiency, profitability, and land utilization index of the sequence at Abeokuta in 2011/2012 cropping season.

\begin{tabular}{|c|c|c|c|c|c|c|c|c|c|c|}
\hline \multicolumn{3}{|c|}{ Treatments } & \multicolumn{4}{|c|}{ Productivity $\left(\mathrm{t} \mathrm{ha}^{-1}\right)$} & \multirow{2}{*}{$\begin{array}{c}\text { Duration } \\
\text { (days) }\end{array}$} & \multirow{2}{*}{$\begin{array}{c}\text { Production } \\
\text { efficiency } \\
\left(\mathrm{kg} \mathrm{ha}^{-1} \mathrm{day}^{-1}\right)\end{array}$} & \multirow{2}{*}{$\begin{array}{l}\text { Profitability } \mathrm{N} \\
\mathrm{ha}^{-1} \mathrm{day}^{-1} \text { ) }\end{array}$} & \multirow{2}{*}{$\begin{array}{c}\text { Land } \\
\text { utilization } \\
\text { index }(\%)\end{array}$} \\
\hline SOY & LR & UR & RGEY & $\begin{array}{c}\text { MRE } \\
\text { LR }\end{array}$ & $\begin{array}{l}\text { MRE } \\
\text { UR }\end{array}$ & TOTAL & & & & \\
\hline $\mathrm{T}_{1} \mathrm{~V}_{1} \mathrm{R}_{1}$ & $\mathrm{~T}_{1} \mathrm{~S}_{1} \mathrm{R}_{1}$ & $\mathrm{~T}_{1} \mathrm{~S}_{1} \mathrm{R}_{1}$ & 0.71 & 2.27 & 1.29 & 4.27 & 303.3 & 14.08 & 1348 & 83.11 \\
\hline $\mathrm{T}_{1} \mathrm{~V}_{1} \mathrm{R}_{2}$ & $\mathrm{~T}_{1} \mathrm{~S}_{1} \mathrm{R}_{2}$ & $\mathrm{~T}_{1} \mathrm{~S}_{1} \mathrm{R}_{2}$ & 0.65 & 2.28 & 1.10 & 4.03 & 283.3 & 14.21 & 1178 & 77.63 \\
\hline $\mathrm{T}_{1} \mathrm{~V}_{1} \mathrm{R}_{3}$ & $\mathrm{~T}_{1} \mathrm{~S}_{1} \mathrm{R}_{3}$ & $\mathrm{~T}_{1} \mathrm{~S}_{1} \mathrm{R}_{3}$ & 0.71 & 2.48 & 1.22 & 4.41 & 302.0 & 14.60 & 1404 & 82.74 \\
\hline $\mathrm{T}_{1} \mathrm{~V}_{2} \mathrm{R}_{1}$ & $\mathrm{~T}_{1} \mathrm{~S}_{2} \mathrm{R}_{1}$ & $\mathrm{~T}_{1} \mathrm{~S}_{2} \mathrm{R}_{1}$ & 0.67 & 2.31 & 1.33 & 4.30 & 290.0 & 14.84 & 1345 & 79.45 \\
\hline $\mathrm{T}_{1} \mathrm{~V}_{2} \mathrm{R}_{2}$ & $\mathrm{~T}_{1} \mathrm{~S}_{2} \mathrm{R}_{2}$ & $\mathrm{~T}_{1} \mathrm{~S}_{2} \mathrm{R}_{2}$ & 0.72 & 2.16 & 1.16 & 4.03 & 303.0 & 13.31 & 1195 & 83.01 \\
\hline $\mathrm{T}_{1} \mathrm{~V}_{2} \mathrm{R}_{3}$ & $\mathrm{~T}_{1} \mathrm{~S}_{2} \mathrm{R}_{3}$ & $\mathrm{~T}_{1} \mathrm{~S}_{2} \mathrm{R}_{3}$ & 0.67 & 2.34 & 1.16 & 4.16 & 292.3 & 14.24 & 1248 & 80.09 \\
\hline $\mathrm{T}_{2} \mathrm{~V}_{1} \mathrm{R}_{1}$ & $\mathrm{~T}_{2} \mathrm{~S}_{1} \mathrm{R}_{1}$ & $\mathrm{~T}_{2} \mathrm{~S}_{1} \mathrm{R}_{1}$ & 0.62 & 2.12 & 1.25 & 3.99 & 289.3 & 13.78 & 1081 & 79.27 \\
\hline $\mathrm{T}_{2} \mathrm{~V}_{1} \mathrm{R}_{2}$ & $\mathrm{~T}_{2} \mathrm{~S}_{1} \mathrm{R}_{2}$ & $\mathrm{~T}_{2} \mathrm{~S}_{1} \mathrm{R}_{2}$ & 0.70 & 2.34 & 1.44 & 4.48 & 302.0 & 14.83 & 1379 & 82.74 \\
\hline $\mathrm{T}_{2} \mathrm{~V}_{1} \mathrm{R}_{3}$ & $\mathrm{~T}_{2} \mathrm{~S}_{1} \mathrm{R}_{3}$ & $\mathrm{~T}_{2} \mathrm{~S}_{1} \mathrm{R}_{3}$ & 0.63 & 2.50 & 1.45 & 4.57 & 291.3 & 15.69 & 1400 & 79.82 \\
\hline $\mathrm{T}_{2} \mathrm{~V}_{2} \mathrm{R}_{1}$ & $\mathrm{~T}_{2} \mathrm{~S}_{2} \mathrm{R}_{1}$ & $\mathrm{~T}_{2} \mathrm{~S}_{2} \mathrm{R}_{1}$ & 0.68 & 2.16 & 1.47 & 4.31 & 303.7 & 14.18 & 1261 & 83.20 \\
\hline $\mathrm{T}_{2} \mathrm{~V}_{2} \mathrm{R}_{2}$ & $\mathrm{~T}_{2} \mathrm{~S}_{2} \mathrm{R}_{2}$ & $\mathrm{~T}_{2} \mathrm{~S}_{2} \mathrm{R}_{2}$ & 0.63 & 2.34 & 1.43 & 4.40 & 289.3 & 15.21 & 1315 & 79.27 \\
\hline $\mathrm{T}_{2} \mathrm{~V}_{2} \mathrm{R}_{3}$ & $\mathrm{~T}_{2} \mathrm{~S}_{2} \mathrm{R}_{3}$ & $\mathrm{~T}_{2} \mathrm{~S}_{2} \mathrm{R}_{3}$ & 0.68 & 2.46 & 1.29 & 4.43 & 300.3 & 14.76 & 1340 & 82.28 \\
\hline \multirow[t]{2}{*}{ Tel. (F) } & $\begin{array}{c}\mathrm{T}_{2} \mathrm{~S}_{2} \mathrm{R}_{2} \\
(\mathrm{~F})\end{array}$ & $\begin{array}{c}\mathrm{T}_{2} \mathrm{~S}_{2} \mathrm{R}_{2} \\
(\mathrm{~F})\end{array}$ & 0.99 & 1.69 & 1.01 & 3.69 & 323.3 & 11.40 & 729 & 88.58 \\
\hline & $\operatorname{LSD}(0.05)$ & & 0.078 & 0.47 & 0.33 & 0.59 & 6.611 & 1.911 & 327.9 & 1.811 \\
\hline
\end{tabular}

Legend: $\mathrm{T}$ - tillage: $\mathrm{T}_{1}-$ minimum, $\mathrm{T}_{2}$ - conventional; $\mathrm{V}$ - soybean varieties: $\mathrm{V}_{1}-\mathrm{TGx}$ 1448-2E, $\mathrm{V}_{2}-\mathrm{TGx} 1740-2 \mathrm{~F}$; $\mathrm{R}-$ seeding rates: for soybean $=R_{1}-60 \mathrm{~cm} \mathrm{X} 5 \mathrm{~cm}, R_{2}-60 \mathrm{~cm} \mathrm{X} 10 \mathrm{~cm}, R_{3}-60 \mathrm{~cm} \mathrm{X} 15 \mathrm{~cm}$; For rice $=R_{1}-15 \mathrm{~cm} \mathrm{X} 15 \mathrm{~cm}, R_{2}-20 \mathrm{~cm} \mathrm{X} 20 \mathrm{~cm}, R_{3}-25 \mathrm{~cm} \mathrm{X} 25$ $\mathrm{cm} ; \mathrm{S}$ - seeding method: $\mathrm{S}_{1}$ - dry dibble, $\mathrm{S}_{2}$ - transplanting; $\mathrm{F}$ - inorganic fertilizer, Tel. - Telfairia occidentalis (fluted pumpkin), SOY - soybean,

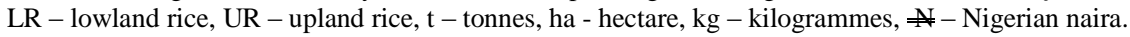


Yield response and economic implications of soybean ... cropping in the rainforest/savanna transitory ecosystem

Table 11: Total productivity, duration, production efficiency, profitability, and land utilization index of the sequence at Ibadan in 2011/2012 cropping season.

\begin{tabular}{|c|c|c|c|c|c|c|c|c|c|c|}
\hline \multicolumn{3}{|c|}{ Treatments } & \multicolumn{4}{|c|}{ Productivity $\left(\mathrm{t} \mathrm{ha}^{-1}\right)$} & \multirow{2}{*}{$\begin{array}{c}\begin{array}{c}\text { Duration } \\
\text { (days) }\end{array}\end{array}$} & \multirow{2}{*}{$\begin{array}{l}\text { Production } \\
\text { efficiency } \\
\left(\mathrm{kg} \mathrm{ha}^{-1} \text { day }^{-1}\right)\end{array}$} & \multirow{2}{*}{ 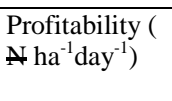 } & \multirow{2}{*}{$\begin{array}{c}\text { Land } \\
\text { utilization } \\
\text { index }(\%)\end{array}$} \\
\hline SOY & LR & UR & RGEY & $\begin{array}{c}\text { MRE } \\
\text { LR }\end{array}$ & $\begin{array}{l}\text { MRE } \\
\text { UR }\end{array}$ & TOTAL & & & & \\
\hline $\mathrm{T}_{1} \mathrm{~V}_{1} \mathrm{R}_{1}$ & $\mathrm{~T}_{1} \mathrm{~S}_{1} \mathrm{R}_{1}$ & $\mathrm{~T}_{1} \mathrm{~S}_{1} \mathrm{R}_{1}$ & 0.50 & 2.42 & 1.69 & 4.60 & 300.7 & 15.30 & 1531 & 82.37 \\
\hline $\mathrm{T}_{1} \mathrm{~V}_{1} \mathrm{R}_{2}$ & $\mathrm{~T}_{1} \mathrm{~S}_{1} \mathrm{R}_{2}$ & $\mathrm{~T}_{1} \mathrm{~S}_{1} \mathrm{R}_{2}$ & 0.63 & 2.12 & 1.26 & 4.01 & 283.3 & 14.13 & 1166 & 77.63 \\
\hline $\mathrm{T}_{1} \mathrm{~V}_{1} \mathrm{R}_{3}$ & $\mathrm{~T}_{1} \mathrm{~S}_{1} \mathrm{R}_{3}$ & $\mathrm{~T}_{1} \mathrm{~S}_{1} \mathrm{R}_{3}$ & 0.63 & 2.20 & 1.37 & 4.19 & 300.7 & 13.93 & 1281 & 82.37 \\
\hline $\mathrm{T}_{1} \mathrm{~V}_{2} \mathrm{R}_{1}$ & $\mathrm{~T}_{1} \mathrm{~S}_{2} \mathrm{R}_{1}$ & $\mathrm{~T}_{1} \mathrm{~S}_{2} \mathrm{R}_{1}$ & 0.58 & 2.47 & 1.40 & 4.45 & 290.3 & 15.33 & 1427 & 79.54 \\
\hline $\mathrm{T}_{1} \mathrm{~V}_{2} \mathrm{R}_{2}$ & $\mathrm{~T}_{1} \mathrm{~S}_{2} \mathrm{R}_{2}$ & $\mathrm{~T}_{1} \mathrm{~S}_{2} \mathrm{R}_{2}$ & 0.68 & 1.92 & 1.09 & 3.69 & 303.0 & 12.19 & 1005 & 83.01 \\
\hline $\mathrm{T}_{1} \mathrm{~V}_{2} \mathrm{R}_{3}$ & $\mathrm{~T}_{1} \mathrm{~S}_{2} \mathrm{R}_{3}$ & $\mathrm{~T}_{1} \mathrm{~S}_{2} \mathrm{R}_{3}$ & 0.59 & 2.10 & 1.11 & 3.79 & 290.3 & 13.07 & 1040 & 79.54 \\
\hline $\mathrm{T}_{2} \mathrm{~V}_{1} \mathrm{R}_{1}$ & $\mathrm{~T}_{2} \mathrm{~S}_{1} \mathrm{R}_{1}$ & $\mathrm{~T}_{2} \mathrm{~S}_{1} \mathrm{R}_{1}$ & 0.53 & 2.47 & 1.43 & 4.43 & 289.0 & 15.32 & 1327 & 79.18 \\
\hline $\mathrm{T}_{2} \mathrm{~V}_{1} \mathrm{R}_{2}$ & $\mathrm{~T}_{2} \mathrm{~S}_{1} \mathrm{R}_{2}$ & $\mathrm{~T}_{2} \mathrm{~S}_{1} \mathrm{R}_{2}$ & 0.61 & 2.61 & 1.20 & 4.42 & 300.7 & 14.70 & 1347 & 82.37 \\
\hline $\mathrm{T}_{2} \mathrm{~V}_{1} \mathrm{R}_{3}$ & $\mathrm{~T}_{2} \mathrm{~S}_{1} \mathrm{R}_{3}$ & $\mathrm{~T}_{2} \mathrm{~S}_{1} \mathrm{R}_{3}$ & 0.60 & 2.26 & 1.26 & 4.12 & 290.7 & 14.17 & 1149 & 79.63 \\
\hline $\mathrm{T}_{2} \mathrm{~V}_{2} \mathrm{R}_{1}$ & $\mathrm{~T}_{2} \mathrm{~S}_{2} \mathrm{R}_{1}$ & $\mathrm{~T}_{2} \mathrm{~S}_{2} \mathrm{R}_{1}$ & 0.61 & 2.10 & 1.11 & 3.81 & 302.3 & 12.61 & 988 & 82.83 \\
\hline $\mathrm{T}_{2} \mathrm{~V}_{2} \mathrm{R}_{2}$ & $\mathrm{~T}_{2} \mathrm{~S}_{2} \mathrm{R}_{2}$ & $\mathrm{~T}_{2} \mathrm{~S}_{2} \mathrm{R}_{2}$ & 0.53 & 2.33 & 1.30 & 4.16 & 288.0 & 14.46 & 1182 & 78.90 \\
\hline $\mathrm{T}_{2} \mathrm{~V}_{2} \mathrm{R}_{3}$ & $\mathrm{~T}_{2} \mathrm{~S}_{2} \mathrm{R}_{3}$ & $\mathrm{~T}_{2} \mathrm{~S}_{2} \mathrm{R}_{3}$ & 0.73 & 2.70 & 1.32 & 4.75 & 300.3 & 15.84 & 1520 & 82.28 \\
\hline \multirow[t]{2}{*}{ Tel. (F) } & $\begin{array}{l}\mathrm{T}_{2} \mathrm{~S}_{2} \mathrm{R}_{2} \\
(\mathrm{~F})\end{array}$ & $\begin{array}{c}\mathrm{T}_{2} \mathrm{~S}_{2} \mathrm{R}_{2} \\
(\mathrm{~F})\end{array}$ & 1.04 & 1.70 & 0.96 & 3.70 & 323.3 & 11.43 & 733 & 88.58 \\
\hline & $\operatorname{LSD}(0.05)$ & & 0.20 & 0.64 & 0.34 & 0.80 & 7.53 & 2.74 & 444.3 & 2.06 \\
\hline
\end{tabular}

Table 12: Economics of the rice-based cropping sequence in Abeokuta and Ibadan in 2011/2012 cropping season.

\begin{tabular}{|c|c|c|c|c|c|c|c|c|c|c|c|c|c|}
\hline \multicolumn{3}{|c|}{ Treatments } & \multicolumn{2}{|c|}{$\begin{array}{c}\text { Total } \\
\text { productivity } \\
\left(\mathrm{t} \mathrm{ha}^{-1}\right)\end{array}$} & \multirow[t]{2}{*}{$\begin{array}{l}\text { Cost of } \\
\text { Cultivation } \\
\left(\mathrm{N} \mathrm{ha}^{-1}\right)\end{array}$} & \multicolumn{2}{|c|}{$\begin{array}{l}\text { Gross Return ( } \$ \text { ha }^{-} \\
\left.{ }^{1}\right)\end{array}$} & \multicolumn{2}{|c|}{$\begin{array}{l}\text { Net Return }(\mathrm{N} \\
\left.\mathrm{ha}^{-1}\right)\end{array}$} & \multicolumn{2}{|c|}{$\begin{array}{l}\text { Benefit: Cost } \\
\text { Ratio }\end{array}$} & \multicolumn{2}{|c|}{$\begin{array}{c}\text { Economic } \\
\text { Efficiency (ha } \\
\left.1 \text { day }^{-1}\right)\end{array}$} \\
\hline SOY & LR & UR & $\mathrm{Ab}$. & Ib. & & Ab. & Ib. & $\mathrm{Ab}$. & Ib. & $\mathrm{Ab}$ & Ib. & $\mathrm{Ab}$ & Ib. \\
\hline $\mathrm{T}_{1} \mathrm{~V}_{1} \mathrm{R}_{1}$ & $\mathrm{~T}_{1} \mathrm{~S}_{1} \mathrm{R}_{1}$ & $\mathrm{~T}_{1} \mathrm{~S}_{1} \mathrm{R}_{1}$ & 4.27 & 4.60 &, 100 & 864048 & 30837 & 491858 & 558647 & 1.32 & 1.50 & 1621 & 1858 \\
\hline $\mathrm{T}_{1} \mathrm{~V}_{1} \mathrm{R}_{2}$ & $\mathrm{~T}_{1} \mathrm{~S}_{1} \mathrm{R}_{2}$ & $\mathrm{~T}_{1} \mathrm{~S}_{1} \mathrm{R}_{2}$ & 3 & 4.01 & 0 & 16 & 3 & 429836 & 425 & 1.12 & 1.11 & 514 & 1498 \\
\hline $\mathrm{T}_{1} \mathrm{~V}_{1} \mathrm{R}_{3}$ & $\mathrm{~T}_{1} \mathrm{~S}_{1} \mathrm{R}_{3}$ & $\mathrm{~T}_{1} \mathrm{~S}_{1}$ & 1 & 4. & 0 & 68 & 8 & 5 & 4 & 1.35 & 1. & 696 & 1556 \\
\hline $\mathrm{T}_{1} \mathrm{~V}_{2} \mathrm{R}_{1}$ & $\mathrm{~T}_{1} \mathrm{~S}_{2} \mathrm{R}_{1}$ & $\mathrm{~T}_{1} \mathrm{~S}_{2}$ & 0 & 4. & 00 & 870753 & 499 & 491063 & 520809 & 1.29 & 1.37 & 1693 & 1794 \\
\hline $\mathrm{T}_{1} \mathrm{~V}_{2} \mathrm{R}_{2}$ & $\mathrm{~T}_{1} \mathrm{~S}_{2} \mathrm{R}_{2}$ & $\mathrm{~T}_{1} \mathrm{~S}_{2} \mathrm{R}_{2}$ & 4.03 & 3.69 & 880 & 816186 & 747035 & 436056 & 366905 & 1.15 & 0.96 & 1439 & 1213 \\
\hline $\mathrm{T}_{1} \mathrm{~V}_{2} \mathrm{R}_{3}$ & $\mathrm{~T}_{1} \mathrm{~S}_{2} \mathrm{R}_{3}$ & $\mathrm{~T}_{1} \mathrm{~S}_{2} \mathrm{R}_{3}$ & 4.16 & 3.79 & 379,690 & 842621 & 766752 & 455521 & 379652 & 1.18 & 0.98 & 1558 & 1310 \\
\hline $\mathrm{T}_{2} \mathrm{~V}_{1} \mathrm{R}_{1}$ & $\mathrm{~T}_{2} \mathrm{~S}_{1} \mathrm{R}_{1}$ & $\mathrm{~T}_{2} \mathrm{~S}_{1} \mathrm{R}_{1}$ & 3.99 & 4.43 & 411,100 & 806486 & 896062 & 394606 & 484182 & 0.96 & 1.18 & 1364 & 1675 \\
\hline $\mathrm{T}_{2} \mathrm{~V}_{1} \mathrm{R}_{2}$ & $\mathrm{~T}_{2} \mathrm{~S}_{1} \mathrm{R}_{2}$ & $\mathrm{~T}_{2} \mathrm{~S}_{1} \mathrm{R}_{2}$ & 4.48 & 4.42 & 406,780 & 906331 & 894434 & 503391 & 491494 & 1.25 & 1.22 & 1666 & 1633 \\
\hline $\mathrm{T}_{2} \mathrm{~V}_{1} \mathrm{R}_{3}$ & $\mathrm{~T}_{2} \mathrm{~S}_{1} \mathrm{R}_{3}$ & $\mathrm{~T}_{2} \mathrm{~S}_{1} \mathrm{R}_{3}$ & 4.57 & 4.1 & 2,940 & 925130 & 833661 & 511030 & 419561 & 1.2 & $1.01 \mathrm{~b}$ & 1754 & 1442 \\
\hline $\mathrm{T}_{2} \mathrm{~V}_{2} \mathrm{R}_{1}$ & $\mathrm{~T}_{2} \mathrm{~S}_{2} \mathrm{R}_{1}$ & $\mathrm{~T}_{2} \mathrm{~S}_{2} \mathrm{R}$ & 4.31 & 3.8 & 4 & 6 & 71786 & 460 & 360686 & 1.12 & 0.8 & 516 & 1193 \\
\hline $\mathrm{T}_{2} \mathrm{~V}_{2} \mathrm{R}_{2}$ & $\mathrm{~T}_{2} \mathrm{~S}_{2} \mathrm{R}_{2}$ & $\mathrm{~T}_{2} \mathrm{~S}_{2} \mathrm{R}$ & 4.40 & 4.16 & 4 & 89 & 35 & 4795 & 431 & 1.1 & 1.0 & 658 & 1500 \\
\hline $\mathrm{T}_{2} \mathrm{~V}_{2} \mathrm{R}_{3}$ & $\mathrm{~T}_{2} \mathrm{~S}_{2} \mathrm{R}_{3}$ & $\mathrm{~T}_{2} \mathrm{~S}_{2} \mathrm{R}_{3}$ & 4.43 & 4.75 & 410,440 & 895 & 961448 & 489173 & 554668 & 1.20 & 1.36 & 1632 & 1850 \\
\hline Tel. (F) & $\begin{array}{l}\mathrm{T}_{2} \mathrm{~S}_{2} \mathrm{R}_{2} \\
(\mathrm{~F})\end{array}$ & $\begin{array}{l}\mathrm{T}_{2} \mathrm{~S}_{2} \mathrm{R}_{2} \\
(\mathrm{~F})\end{array}$ & 3.69 & 3.70 & 100 & 746093 & 747712 & 265993 & 267612 & 0.55 & 0.56 & 822 & 828 \\
\hline & LSD (0.05) & & 0.59 & 0.80 & 3.8 & 119142.3 & 161979 & 746093 & 162185.2 & 0.298 & 0.412 & 392.4 & 552.5 \\
\hline
\end{tabular}

Legend: $\mathrm{T}$ - Tillage: $\mathrm{T}_{1}-$ Minimum, $\mathrm{T}_{2}-$ Conventional; $\mathrm{V}$ - Soybean varieties: $\mathrm{V}_{1}-\mathrm{TGx}$ 1448-2E, $\mathrm{V}_{2}-\mathrm{TGx}$ 1740-2F; $\mathrm{R}-\mathrm{Seeding}$ rates: for soybean $=R_{1}-60 \mathrm{~cm} \mathrm{X} 5 \mathrm{~cm}, R_{2}-60 \mathrm{~cm} \mathrm{X} 10 \mathrm{~cm}, R_{3}-60 \mathrm{~cm} \times 15 \mathrm{~cm}$; For rice $=R_{1}-15 \mathrm{~cm} \times 15 \mathrm{~cm}, R_{2}-20 \mathrm{~cm} \times 20 \mathrm{~cm}, R_{3}-25 \mathrm{~cm} \times 25 \mathrm{~cm}$; $\mathrm{S}$ - seeding method: $\mathrm{S}_{1}-$ dry dibble, $\mathrm{S}_{2}-$ transplanting; $\mathrm{F}$ - inorganic fertilizer, Tel. - Telfairia occidentalis (fluted pumpkin), SOY - Soybean, LR

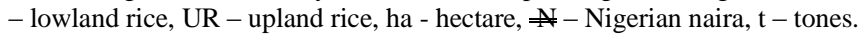

\section{DISCUSSION}

Intensive cropping system through sequential cropping could be constrained by growth factors; selection of appropriate cropping mixture in the right environment could ameliorate the negative effect of increased utilization of growth resources (Malézieux et al., 2009). Available literature had indicated varietal variability on yield components of soybean (Baruah et al., 2014); this was more pronounced in Abeokuta than in Ibadan. This suggests that genotypic differences could have been mediated by environmental factors. Biological nitrogen fixation is an energy consuming process (Serraj et al.,
1999). This process is limited by nutrient elements, especially phosphorus (Harold et al., 1990). In legumes in symbiotic relation with microorganism. Oxidative phosphorylation and availability of reducing compounds such as nicotinamide adenine dinucleotide phosphate (NADP) could be grossly constrained where $\mathrm{P}$ is limiting (Streeter, 1991). This is especially germane in the tropics, where $\mathrm{P}$ is chelated with $\mathrm{Al}$ or $\mathrm{Fe}$, due to the acidic nature of such soils (Sample et al., 1980) Though $\mathrm{P}$ content in both locations could be characterized as low (Agbede, 2009), in absolute terms soil $\mathrm{P}$ content in 
Abeokuta was observed to be higher than in Ibadan at the start of establishing soybean. This could have explained the significant varietal differences in Abeokuta than Ibadan. This varietal variability was also reflected on mass of pod per plot, mass of seed per plot and seed per pod suggesting that these yield components were the ones that contributed predominantly to the grain yield of soybean in Abeokuta (Pande et al., 1985).

Tillage did not have any significant effect on the yield components and performance of the component crops in the sequence except on panicle mass in the lowland rice (Abeokuta) and panicle length in lowland (Ibadan) and upland rice (Abeokuta). Both locations recorded an increase in soil organic matter in the sequence. However, the proportion of increase was more pronounced at Ibadan than Abeokuta. Puddling is a predominant tillage practice under conventional tillage to ensure comparatively higher water balance (low seepage and percolation) (McDonald et al., 2006) through increase in situ water storage capacity, increase in nutrient form, availability and loss (Wade et al., 1998). Suppression of weed infestation had also been observed (Garrity et al., 1992). Conventional tillage is capable of positively affecting the rate of mineralization and decomposition of organic residues incorporated into the soil after each sequence. Increase in those yield components could have suggested release of essential nutrients for assimilate partitioning at the most critical growth stage of rice. However, decomposition and mineralization rate as reflected in soil organic content and nitrogen availability in lowland could have suggested slower rate due to anaerobic soil condition and its implications on the activities of soil microbes. The threefold increase in soil organic matter especially at Ibadan during the establishment of upland rice could be explained by the $\mathrm{pH}$ of the soil which is close to neutral that could hasten microbial activities for organic residue mineralization or the textural composition of the soil preceding upland rice which could positively affect soil nutrient reservoir. Similar pattern were observed on the dynamics of nitrogen in the soil at both locations.

Increased stover mass per plot observed at both locations with increased soybean density and a depression on 100 grain mass (Abeokuta) and seed mass per pod (Ibadan) suggested a compensatory relationship between vegetative and reproductive growth. With increased plant density there would be increased competition for growth resources and metabolite that must be optimized for maximum grain yield for a given genotype in a given environment. At both locations most yield components of lowland rice increased with a reduction in plant density, except number of panicle per $\mathrm{m}^{2}$ (both locations) and 1000 seed mass (Ibadan). Total nitrogen at both locations was classified as low in Nigeria according to Agbede, (2009), incorporation of soybean residue and the application of organic manure, which are slow releasing with the physico-chemical changes reported in lowland ecology (Ponnamperuma, 1972), could have limited availability of essential nutrients, especially nitrogen at both locations. Inland valley are characterized by a predominance of reduction reaction (reduced soil $\mathrm{pH}$ and redox potential) with concomitant effect on nutrient availability, microbial activity and changes in structural integrity of soil particles (Ponnamperuma, 1972). In lowland rice increased number of panicles per $\mathrm{m}^{2}$ at both locations and 1000 grain mass (Ibadan) with increasing plant densities could have resulted from the increased number of tillers per $\mathrm{m}^{2}$, increased number of tillers per hill and or increased hill per $\mathrm{m}^{2}$ (Huang et al., 2011). Huang et al., (2011) posited that number of tillers per hill are determined by the tillering rate and duration and metabolic process (nitrogen and carbon land assimilation). Rainfall distribution at Ibadan in June, 2011 was more than what was obtained in Abeokuta, which could have contributed more to the relative better performance of yield components observed at Ibadan with increasing plant density. Upland rice had all its yield components increased with decreasing plant densities at both locations. This could have suggested that preceding lowland rice, with altered physicochemical soil condition in a submerged soil could have depleted nutrient availability; hence increasing succeeding upland rice plant population in the inland valley would have exacerbated increased competition for nutrients in the soil.

Most yield components of transplanted lowland rice recorded better performance than dry dibble method in both locations, though not significant, except significantly higher number of grains per panicle that was achieved in Abeokuta under dry dibble method than transplanting. Earlier reported literature indicated that dry dibble method increased number of panicle per $\mathrm{m}^{2}$, which increased the sink strength (Huang et al., 2011). But increased panicle sterility that was observed in dry dibble could have compromised its comparatively superior performance in the lowland rice (Farooq et al., 2011). Other factors reported included the prevalence of weed, reduced water use efficiency and nutrient availability (Farooq et al., 2011). The trend in the upland rice was that dry dibble gave better yield components than transplanted method especially on number of panicle per $\mathrm{m}^{2}$ and grain yield (Abeokuta) and number of grains per panicle (Ibadan). Other reports had reported comparatively similar yield of dry dibble and transplanted seeding method (Kukal and Aggarwal, 2002). This could have been a result of its positive effect on yield components mediated by environmental conditions. 
The high production efficiency in Abeokuta for the sequence soybean 'TGx 144-2E', spaced at $60 \mathrm{~cm} \times 15$ $\mathrm{cm}$, conventionally tilled, with lowland and upland rice conventionally tilled and directly seeded at the spacing of $25 \mathrm{~cm} \times 25 \mathrm{~cm}$ could be as a result of its high total productivity, explained by the high productivity of milled lowland rice. This information is corroborated by earlier studies that indicated a comparatively higher performance of lowland than upland rice in most rice growing ecologies (Grist, 1986). However, reduced land use index (LUI) observed in the sequence soybean 'TGx $144-2 \mathrm{E}$ ', spaced at $60 \mathrm{~cm} \times 10 \mathrm{~cm}$, minimally tilled with lowland and upland rice minimally tilled and directly seeded at the spacing $20 \mathrm{~cm} \times 20 \mathrm{~cm}$ could be as a result of a reduced duration of the sequence in a year. Similar pattern was equally observed at Ibadan. This suggests that the sequence was not able to optimally utilize available growth resources. It had been reported that directly seeded rice flower earlier than transplanted (Santhi et al., 1998), which could have explained the short growing season observed. In Ibadan high production efficiency was recorded in the sequence soybean 'TGx 1740-2F', planted at a spacing of $60 \mathrm{~cm}$ $\times 15 \mathrm{~cm}$, conventionally tilled with lowland and upland cost irice conventionally tilled and transplanted at a spacing of $25 \mathrm{~cm} \times 25 \mathrm{~cm}$. Similar pattern with respect to total productivity and productivity of milled lowland rice as observed in Abeokuta was reported here. In Abeokuta, the economic efficiency in the sequence that consisted of soybean variety TGx 1448-2E, spaced at 60 $\mathrm{cm} \times 15 \mathrm{~cm}$, conventionally tilled, lowland and upland rice conventionally tilled and directly seeded at the spacing $25 \mathrm{~cm} \times 25 \mathrm{~cm}$ could have been a result of its high gross returns and high total productivity. However, at Abeokuta high profitability of the sequence soybean variety TGx $1448-2 \mathrm{E}$, spaced at $60 \mathrm{~cm} \times 15 \mathrm{~cm}$, minimally tilled with lowland and upland rice minimally tilled and directly seeded spaced at $25 \mathrm{~cm} \times$ $25 \mathrm{~cm}$ indicated its high benefit-to-cost ratio. In Ibadan, high economic efficiency was observed in the sequence of soybean variety TGx $1448-2 \mathrm{E}$, spaced at $60 \mathrm{~cm} \times$ $5 \mathrm{~cm}$, minimally tilled, with lowland and upland rice minimally tilled and directly seeded, spaced at $15 \mathrm{~cm} \times$ $15 \mathrm{~cm}$. High benefit-to-cost ratio and net return would have explained this higher economic efficiency observed at Ibadan. This was also reflected on its profitability of that sequence.

\section{CONCLUSION}

In Abeokuta, lowland rice had significantly higher panicle mass $(21.44 \mathrm{~g})$ with conventional than minimum tillage, while at Ibadan significantly longer panicle $(25.03 \mathrm{~g})$ was observed. Significantly longer panicle $(22.36 \mathrm{~g})$ was obtained at Abeokuta for upland rice under conventional than minimum tillage. Lowland rice had significantly higher number of grains panicle $^{-1}$ under dry dibble than transplanted rice (Abeokuta). Upland rice had significantly higher number of panicles $\mathrm{m}^{-2}$ and grain yield under dry dibble than transplanted rice (Abeokuta). However, at Ibadan significantly higher numbers of grains panicle ${ }^{-1}$ was recorded for dry dibble than transplanted rice. Soybean had significantly higher stover mass plot $^{-1}$ at both locations with spacing, while significant effect was observed on mass of seed pod $^{-1}$ and 100 grain mass (Abeokuta). Number of panicles $\mathrm{m}^{-2}$ increased with higher lowland rice density (both locations) and 1000 seed mass (Ibadan). Conversely panicle mass, number of grains panicle ${ }^{-1}$ and grain yield recorded significant depression with increasing upland rice (Ibadan). In Abeokuta soybean 'TGx 1448-2E' conventionally established at the $60 \mathrm{~cm}$ $\times 15 \mathrm{~cm}$ spacing, lowland and upland rice at a spacing of $25 \mathrm{~cm} \times 25 \mathrm{~cm}$ that were conventionally tilled and directly seeded was the most economically efficient ( $\left.1,754 \mathrm{ha}^{-1} \mathrm{day}^{-1}\right)$. At Ibadan the sequence of 'TGx 1448$2 \mathrm{E}^{\prime}$ soybean at the spacing of $60 \mathrm{~cm} \times 5 \mathrm{~cm}$ and lowland and upland rice at a spacing of $15 \mathrm{~cm} \times 15 \mathrm{~cm}$ that was directly seeded and established under minimum tillage was the most economically efficient ( $\$ 1,858 \mathrm{ha}^{-}$ ${ }^{1}$ day $\left.^{-1}\right)$.

\section{ACKNOWLEDGEMENT}

This project was ably supported by the management and staff of AfricaRice, Ibadan sub-station, especially in the provision of planting materials and implementation of part of the field experiments. 


\section{REFERENCES}

Adigbo, S. O., Ojerinde, A. O., Ajayi, O., and Nwilene, F. E. (2010). Effect of sowing methods on performance of upland rice in lowland rice-upland rice-vegetable sequence in inland valley. Journal of Agricultural science and technology, 4(3), 1-10

Agbede, O. (2009). Chemical soil analysis and soil testing. In Understanding Soil and Plant Nutrition (pp. 193-197). Keffi, Nasarawa State, Nigeria: Salman Press and Co, Nigeria Ltd.

Akanbi, W. B., Olaniran, O. A., Olaniyi, J. O., Ojo, M. A., Sanusi, O. O. (2007). Effect of cassava peel compost on growth and nutritional quality of Celosia (Celosia argentea L.). Research Journal of Agronomy, 1(3), 110-115.

Allison, L. (1965). Organic carbon. In C.A Black (Ed.), Methods of soil analysis. Part 2 (pp. 1307-1378). Madison: American Society of Agronomy.

Ampong-Nyarko, K. (1996). Weed management in rice in Africa. In B. Auld \& K. . Kim (Eds.), Weed management in rice (pp. 183-191). Rome: FAO.

Azeez, J. O., Van Averbeke, W. (2010). Fate of manure phosphorus in a weathered sandy clay loam soil amended with three animal manures. Bioresource Technology, 101(16), 6584-6588. doi:10.1016/j.biortech.2010.03.073

Baruah, S., Sarma, M., Baishya, D., Sharma, A. ., Borah, R., Bhuyan, J. (2014). Genetic variation for seed yield and yellow mosaic virus resistance in Soybean [Glysine max (L.) Merrill]. International Journal of Scientific and Research Publications, 4(9), 1-10.

Bouyoucos, G. J. (1962). Hydrometer method improved for making particle size analyses of soils. Agronomy Journal, 54(5), 464-465. doi:10.2134/agronj1962.00021962005400050028x

Bray, R. ., Kurtz, L. (1945). Determination of total, organic and available forms of phosphorus in soil. Soil Science, 59, 39-45. doi:10.1097/00010694194501000-00006

Chan, C. C., Nor, M. A. M. (1993). Impacts and implications of direct seeding on irrigation requirement and systems management. In Workshop on Water and Direct Seeding for Rice (pp. 14-16).

Chidumayo, E. N. (1987). A shifting cultivation land use system under population pressure in Zambia. Agroforestry Systems, 5(1), 15-25. doi.org/10.1007/BF00046411
Daramola, B. (2005). Government policies and competitiveness of Nigerian rice economy. Workshop on Rice Policy and Food Security in sub-Sahara Africa, Cotonou, Republic of Benin.

Farooq, M., Siddique, K. H., Rehman, H., Aziz, T., Lee, D.J., Wahid, A. (2011). Rice direct seeding: experiences, challenges and opportunities. Soil and Tillage Research, 111(2), 87-98. doi:10.1016/j.still.2010.10.008

Farooq, M., Wahid, A., Lee, D.J., Ito, O., Siddique, K. H. (2009). Advances in drought resistance of rice. Critical Reviews in Plant Sciences, 28(4), 199-217. doi:10.1080/07352680902952173

Fischer, A. J., Antigua, G. (1996). Weed management for rice in Latin America and the Caribbean. FAO Plant production and protection papers, 157-158.

Garrity, D. P., Movillon, M., Moody, K. (1992). Differential weed suppression ability in upland rice cultivars. Agronomy Journal, 84(4), 586-591. doi:10.2134/agronj1992.00021962008400040009x

Gill, M. S., Kumar, P., Kumar, A. (2006). Growth and yield of direct-seeded rice (Oryza sativa) as influenced by seeding technique and seed rate under irrigated conditions. Indian Journal of Agronomy, 51(4), 283-287.

Grist, D. (1986). Rice (6th ed.). London: Longman Group Ltd.

Harold, H., Keyser, Fudi, L. (1990). Potantial for increasing biological nitrogen fixation in soybean. In J. . Ladha, T. George, \& C. Bohloot (Eds.), Biological nitrogen fixation for sustainable agriculture (pp. 119-135). Netherlands: Springer.

Huang, M., Zou, Y., Jiang, P., Xia, B., Feng, Y., Cheng, Z., Mo, Y. (2011). Yield component differences between direct-seeded and transplanted super hybrid rice. Plant Production Science, 14(4), 331338. doi:10.1626/pps.14.331

Jackson, M. (1962). Soil chemical analysis. New Delhi: Prentice Hall of India Pvt, Ltd.

Kukal, S. S., Aggarwal, G. C. (2002). Percolation losses of water in relation to puddling intensity and depth in a sandy loam rice (Oryza sativa) field. Agricultural Water Management, 57(1), 49-59. doi:10.1016/S0378-3774(02)00037-9

Malézieux, E., Crozat, Y., Dupraz, C., Laurans, M., Makowski, D., Ozier-Lafontaine, H., ValantinMorison, M. (2009). Mixing plant species in cropping systems: concepts, tools and models: a 
review. In Sustainable Agriculture (pp. 329-353). Springer. doi:10.1007/978-90-481-2666-8_22

Manjunath, B. ., Korikanthimah, V. (2004). Productivity under rice-based cropping systems and physicochemical properties of soil as influenced by sources of manure in coastal eco-system of Goa. Journal Farming Research and Development, 10(1\&2), 3340.

Mashingaidze, A. B. (2004). Improving weed management and crop productivity in maize systems in Zimbabwe. Wageningen University and Research Centre.

McDonald, A. J., Riha, S. J., Duxbury, J. M., Steenhuis, T. S., Lauren, J. G. (2006). Water balance and rice growth responses to direct seeding, deep tillage, and landscape placement: Findings from a valley terrace in Nepal. Field Crops Research, 95(2), 367382. doi:10.1016/j.fcr.2005.04.006

McLean, E. O. (1982). Soil $\mathrm{pH}$ and lime requirement. Methods of Soil Analysis. Part 2. Chemical and Microbiological Properties, (methodsofsoilan2), 199-224.

Morris, R. A. (1990). Tillage and seeding methods for dry seeded rice. In Report on Workshop on Cropping Systems Research in Asia (pp. 3-7).

National Cereals Research Institute. (1992). Rice harvesting operation. In Rice production and processing technology (pp. 97-105). Badeggi, Bida, Niger State: NCRI.

Pande, N. C., Samantaray, R. N., Mohanty, S. K. (1985). Nutrient changes in direct-seeded submerged rice soils with varying nutrioenvironments. Plant and Soil, 88(2), 299-306. doi:10.1007/BF02182459

Patil, E. N., Jawale, S. M., Mahajan, M. S. (1995). Production potential, economics and fertility status of soil as influenced by wheat (Triticum aestivum)based cropping system. Indian Journal of Agronomy, 40(4), 544-548.
Ponnamperuma, F. N. (1972). The chemistry of submerged soils (Vol. 24). Academic Press New York. doi:10.1016/S0065-2113(08)60633-1

Prasad, D., Urkurkar, S. ., Narendra, N. (2011). Employment generation and their efficacy of various rice (Oryza sativa) based cropping systems. Research Journal of Agricultural Sciences, 2(1), 79-82.

Rao, A. N., Johnson, D. E., Sivaprasad, B., Ladha, J. K., Mortimer, A. M. (2007). Weed management in direct-seeded rice. Advances in Agronomy, 93, 153255. doi:10.1016/S0065-2113(06)93004-1

Sample, E. C., Soper, R. J., Racz, G. J. (1980). Reactions of phosphate fertilizers in soils. The Role of Phosphorus in Agriculture, (theroleofphosph), 263-310.

Santhi, P., Ponnuswamy, K., Kempu Chetty, N. (1998). Effect of seeding methods and efficient nitrogen management practices on the growth of lowland rice. Journal of Ecobiology, 10, 123-132.

Serraj, R., Sinclair, T. R., Purcell, L. C. (1999). Symbiotic N2 fixation response to drought. Journal of Experimental Botany, 50(331), 143-155. doi: $10.1093 / \mathrm{j} \times \mathrm{b} / 50.331 .143$

Streeter, J. G. (1991). Transport and metabolism of carbon and nitrogen in legume nodules. Academic Press. doi:10.1016/S0065-2296(08)60022-1

Tomar, S. S., Tiwari, A. S. (1990). Production potential and economics of different crop sequences. Indian Journal of Agronomy, 35(1-2), 30-35.

Wade, L. J., George, T., Ladha, J. K., Singh, U., Bhuiyan, S. I., Pandey, S. (1998). Opportunities to manipulate nutrient-by-water interactions in rainfed lowland rice systems. Field Crops Research, 56(1), 93-112. doi:10.1016/S0378-4290(97)00142-1

Zimdahl, R. L. (2013). Fundamentals of weed science. Academic Press. 